\title{
Within-Trial Persistence of Learned Behavior as a Dissociable Behavioral Component in Hippocampus- Dependent Memory Tasks: A Potential Postlearning Role of Immature Neurons in the Adult Dentate Gyrus
}

\author{
Alessandro Luchetti, ${ }^{1, *}$ Takuma Yamaguchi (山口拓馬), ${ }^{1, *}$ Masato Uemura, ${ }^{2}$ Glen Yovianto, ${ }^{1}$ Luka Culig, ${ }^{1}$ \\ Ming Yang, ${ }^{1}$ Wei Zhou, ${ }^{1}$ Franziska Oschmann, ${ }^{2}$ MinFeng Lua, ${ }^{1}$ and Ayumu Tashiro (田代 步) ${ }^{1}$
}

https://doi.org/10.1523/ENEURO.0195-21.2021

${ }^{1}$ School of Biological Sciences, Nanyang Technological University, Singapore 308232 and ${ }^{2}$ Kavli Institute for Systems Neuroscience, Norwegian University of Science and Technology, Trondheim 7491, Norway

\begin{abstract}
The term "memory strength" generally refers to how well one remembers something. But more precisely it contains multiple modalities, such as how easily, how accurately, how confidently and how vividly we remember it. In human, these modalities of memory strength are dissociable. In this study, we asked whether we can isolate a behavioral component that is dissociable from others in hippocampus-dependent memory tasks in mice, which potentially reflect a modality of memory strength. Using a virus-mediated inducible method, we ablated immature neurons in the dentate gyrus in mice after we trained the mice with hippocampus-dependent memory tasks normally. In memory retrieval tests, these ablated mice initially showed intact performance. However, the ablated mice ceased learned behavior prematurely within a trial compared with control mice. In addition, the ablated mice showed shorter duration of individual episodes of learned behavior. Both affected behavioral measurements point to persistence of learned behavior. Thus, the effect of the postlearning manipulation showed dissociation between initial performance and persistence of learned behavior. These two behavioral components are likely to reflect different brain functions and be mediated by separate mechanisms, which might represent different modalities of memory strength. These simple dissociable measurements in widely used behavioral paradigms would be useful to understand detailed mechanisms underlying the expression of learned behavior and potentially different modalities of memory strength in mice. We also discuss a potential role that immature neurons in the dentate gyrus may play in persistence of learned behavior.
\end{abstract}

Key words: adult neurogenesis; fear conditioning; hippocampus; spatial memory

\section{Significance Statement}

We use the term "memory strength" both in everyday life and research settings. Memory strength generally means how well we remember something. However, depending on context, its meaning is varied; it can mean how easily, accurately, vividly and/or confidently we remember it. These different modalities of memory strength are generally correlated but is known to be partly independent. In this study, we asked whether such independent or dissociable behavioral components exist in hippocampus-dependent memory tasks in mice. We provide evidence that initial performance and persistence of learned behavior during memory retrieval tests are dissociable in three memory tasks. Such dissociable components would be helpful to study different modalities of memory strength and their underlying mechanisms in mice in future studies. 


\section{Introduction}

The term "memory strength," which generally means how well one remembers something, is used in both everyday life and research settings. However, its exact meaning can be different depending on situations; you may mean how easily you can remember something, how vividly you remember it, how accurately you remember it, how confidently you remember it or all of them (Bjork and Bjork, 1992; Wozniak et al., 1995; Qin et al., 2011). Although these different modalities of memory strength are often correlated, they are at least partially independent (Squire et al., 2007; Qin et al., 2011; Lacy and Stark, 2013). For example, some of our memories are easy to remember but lack details. Other memories might be difficult to remember but, once remembered, are highly vivid and accurate. It is also possible to have high degree of confidence in a memory that turns out to be false. In human studies, these different modalities of memory strength can be dissociated and have been separately studied (Squire et al., 2007; Geib et al., 2017; Sekeres et al., 2018).

With animal experiments, can we study such different aspects of memory strength? Although the lack of methods to study subjective experience limits experimental approaches for some of the modalities, it should be still possible for other modalities by using objective measurements of behavior during memory tasks. In most rodent studies, we examine memory strength by quantifying the amount or strength of learned behavior, which includes how quickly, how long, how often, how persistently, and how correctly the subject shows learned behavior (Morris et al., 1982; Crawley, 2000; Maren, 2001). However, it is rare to consider different aspects of memory strength. This would be partly because most of commonly used parameters in memory tasks are well correlated with each other (Matzel et al., 2003; Maei et al., 2009), and it is not clear whether any of these behavioral measurements represent a specific modality of memory strength and can be dissociable from others. For example, can how fast/accurate mice start learned behavior be dissociated from how persistently they continue it? In this study, we provide positive evidence for this question. We used a virus-mediated method in mice and induced ablation targeting

This work was supported by Ministry of Education, Singapore, Grants 2018T1-002-053, MOE2015-T2-2-035, and MOE2017-T3-1-002; the National Medical Research Council, Ministry of Health, Singapore, Grant NMRC/ OFIRG/0046/2017; the European Research Council Gant 208132; the James S. McDonnell Foundation (A.T.); and a Human Frontier Science Programme long-term fellowship (M.U.).

${ }^{*}$ A.L. and T.Y. contributed equally to this work.

Acknowledgements: We thank Chong Hai Tarng, Chika Yoshii, and Teruyo Tashiro for their technical assistance; other members of the Tashiro lab for comments on this manuscript; Dr. Menno Witter for the use of a confocal microscope; and Dr. Michael Drew and Dr. Dong-oh Seo for sharing behavioral data from their previous work which we consulted to design our protocol.

Correspondence should be addressed to Ayumu Tashiro at atashiro@ ntu.edu.sg.

https://doi.org/10.1523/ENEURO.0195-21.2021

Copyright @ 2021 Luchetti et al.

This is an open-access article distributed under the terms of the Creative Commons Attribution 4.0 International license, which permits unrestricted use, distribution and reproduction in any medium provided that the original work is properly attributed. immature neurons in the dentate gyrus immediately after the mice normally learned hippocampus-dependent memory tasks. We found dissociated effects between simple behavioral measurements reflecting initial performance and persistence of learned behavior.

Original motivation of this study, which is different from the main focus of this report, was to investigate a postlearning role of immature neurons in the adult dentate gyrus. Neurogenesis, the birth of new neurons, persists throughout life in a few regions of the mammalian brain, including the dentate gyrus (Gage, 2000). It has been suggested that newly generated neurons in the adult dentate gyrus play a role in hippocampus-dependent forms of memory (Shors et al., 2001; Snyder et al., 2005; Deng et al., 2009). These new neurons go through a maturational period lasting over a month, during which they exhibit enhanced functional and morphologic plasticity (Wang et al., 2000; Espósito et al., 2005; Zhao et al., 2006; Ge et al., 2007; Tronel et al., 2010; Åmellem et al., 2017). The nature of enhanced plasticity varies between early and late maturational stages (approximately one to three and approximately four to sixweeks, respectively) and has been postulated to create multiple time windows during which the neurons can make unique contributions to memory processes (Aasebø et al., 2011). While new neurons in the late maturational stages have been shown to be involved both during and after memory formation (Denny et al., 2012; Gu et al., 2012), those in the early maturational stages have been shown to contribute to memory formation (Deng et al., 2009; Vukovic et al., 2013; Seo et al., 2015). However, a postlearning role of new neurons at the early maturational stage has not been identified.

\section{Materials and Methods}

\section{Lentiviral vector construction}

A lentiviral transfer vector was designed to express diphtheria toxin (DT) receptor (DTR; Dorland et al., 1979) under the control of the human doublecortin (DCX) promoter (Fig. 1A). cDNA encoding human DTR (pcDNA3 proHB-EGF WT, Addgene) was inserted into a lentiviral transfer plasmid under the control of the human DCX promoter (Karl et al., 2005). DTR is the human homolog of heparin-binding EGF-like growth factor in the cell membrane. DTR has a high affinity for DT, which is a protein synthesis inhibitor produced by Corynebacterium diphtheria, and is the cause of DT sensitivity in human cells (Collier, 1975; Iwamoto et al., 1994; Buch et al., 2005). Because the murine homolog of DTR has a low affinity for DT, murine cells are insensitive to the dose used in this study ( $50 \mathrm{ng} / \mathrm{g}$ body weight). Upon expression of DTR in immature neurons using the lentiviral vector, systemic injection of the dose of DT into mice induces the death of immature neurons (Fig. 1B). To visualize the transduced cells, cDNA encoding enhanced green fluorescent protein (GFP) was inserted $3^{\prime}$ to the DTR gene following an internal ribosome entry site sequence.

Lentiviral particles were produced using a protocol modified from a previously described method (Tashiro et al., 2015b). After the centrifugation steps described in 
Tashiro et al. (2015b), the suspension was purified using Lenti-X Concentrator purification columns (Clontech). Amicon Ultra-4 Centrifugal Filter Units (EMD Millipore) were used to perform buffer exchange to sterile Dulbecco's PBS (DPBS; catalog \#14040-174, Life Technologies) and to concentrate the solution to a final volume of 100-150 $\mu$ l. Aliquots of the lentiviral solution were stored at $-80^{\circ} \mathrm{C}$ until use.

\section{Subjects}

We used male and female C57BL/6 mice bred in our local facility in Norway or Singapore or purchased from Charles River or InVivos. For water maze and fear conditioning tasks, we used female C57BL/6 mice purchased from the two sources above to avoid behavioral variability between two sexes. The mice were 7-12 weeks old at the start of the experiments and were housed in acrylic cages with access to food and water ad libitum under a 12/12 h light/dark cycle. All experiments were approved by the Norwegian Animal Research Authority and/or Institutional animal care and use committee at Nanyang Technological University.

\section{Surgical procedure}

The solution containing the lentiviral vector was stereotaxically injected into the dentate gyrus (Tashiro et al., $2015 b)$. The mice were anesthetized with $5 \%$ isoflurane [2-chloro-2-(difluoromethoxy)-1,1,1-trifluoroethane] in air at a flow rate of $1000 \mathrm{ml} / \mathrm{min}$. The concentration of isoflurane was gradually reduced to $0.75 \%$ or higher, at which concentration deep anesthesia was maintained. The mice were subcutaneously injected with an analgesic $(0.15 \mathrm{~g} / \mathrm{kg} \mathrm{Temgesic})$. For the experiment described in Figure 3 and Figures $5 B-G, 7,8$, a local anesthetic, Marcaine (AstraZeneca) and lignocaine $(1.33 \mathrm{mg} / \mathrm{ml}, 0.1$ $\mathrm{ml}$ ), respectively, was additionally injected subcutaneously above the skull before performing the skin incision. For the experiment described in Figures $5 B-G, 7,8$, buprenorphine $(0.2 \mathrm{ml}, 0.3 \mathrm{mg} / \mathrm{ml})$ was additionally given intraperitoneally before surgery and meloxicam and baytril were additionally given in drinking water $(8$ and $85 \mathrm{mg} / \mathrm{l}$, respectively) for $3 \mathrm{~d}$ after the surgery.

The lentiviral solution was drawn into a microsyringe equipped with a blunt-end 33-gauge needle (Hamilton Company). After the skull was exposed, one or two holes were drilled at the anteroposterior (AP) coordinate: $-1.8 \mathrm{~mm}$; medial-lateral $(\mathrm{ML})$ : $+/-1.8 \mathrm{~mm}$ from the bregma except for the experiment described in Figures $5 B-G, 7,8$, where the coordinate of AP: -2.0 $\mathrm{mm}, \mathrm{ML}:+/-1.3 \mathrm{~mm}$ was used. The tip of the needle was inserted into the brain at the same AP and ML coordinates and lowered to $2.3 \mathrm{~mm}$ ventrally from the skull surface. In each injection, $1.5 \mu \mathrm{l}$ of lentiviral solution was infused at a rate of $2.5 \mathrm{nl} / \mathrm{s}$ (or $3.33 \mathrm{nl} / \mathrm{s}$ for the experiment described in Fig. $5 B-G, 7,8$ ). The titer of the lentiviral solution was $0.1-0.5 \times 10^{5}$ GFP + colony-forming units/ml in HEK 293FT cells (Life Technologies). In the experiments described in Figures $1-3,5 A, H$, the viral vector was injected into one hemisphere. For Figures 2, 3, 5A,H, the other, non-injected hemisphere was used as a control. In the experiments described in
Figure $1 H$, control mice were injected with PBS. In the experiments described in Figures $4,5 B-G, 6-8$, the vector or vehicle was injected into both hemispheres.

\section{DT injection}

DT (Calbiochem) was dissolved in DPBS at a final concentration of $5 \mu \mathrm{g} / \mathrm{ml}$. The mice received a single dose of DT ( $50 \mathrm{ng} / \mathrm{g}$ body weight) intraperitoneally $7 \mathrm{~d}$ after viral injection.

\section{5-Bromo-2'-deoxyuridine (BrdU) injection}

We dissolved BrdU (catalog \#B5002, Sigma) in 0.9\% saline at a concentration of $10 \mathrm{mg} / \mathrm{ml}$, and the solution was filter sterilized. For each mouse, one dose of BrdU (100 $\mu \mathrm{g} / \mathrm{g}$ body weight) was intraperitoneally injected.

\section{Novel environment exploration}

To induce the activation of granule cells in the dentate gyrus, we placed the mice in a novel environment (an open field consisting of a $32 \times 28 \times 28 \mathrm{~cm}$ plastic box with black-striped and white-striped walls). The mice were allowed to explore the novel environment for $15 \mathrm{~min}$. The mice underwent perfusion fixation $90 \mathrm{~min}$ after the novel environment exposure.

\section{Histology}

The preparation of fixed brain sections has been previously described (Tashiro et al., 2015a). Forty-micrometerthick coronal sections were collected from the AP level covering the entire hippocampus ( $\sim 1.22$ to $3.80 \mathrm{~mm}$ posterior to the bregma). The sections were stored in a cryoprotectant solution (20\% glycerin, $30 \%$ ethylene glycol in $0.1 \mathrm{M} \mathrm{PB}$ ) at $-20^{\circ} \mathrm{C}$ until use. After being rinsed with $0.1 \mathrm{~m}$ Tris-buffered saline (TBS), the sections were incubated with a blocking solution [0.25\% Triton X-100 (Sigma) and $3 \%$ donkey serum (Sigma) in $0.1 \mathrm{~m}$ TBS] for $60 \mathrm{~min}$ to minimize non-specific immunoreactions. The sections were incubated with primary antibodies in the blocking solution for $2 \mathrm{~d}$ at $4^{\circ} \mathrm{C}$. Unbound primary antibodies were removed by washing with $0.1 \mathrm{~m}$ TBS, and the sections were then incubated with secondary antibodies in the blocking solution for $4 \mathrm{~h}$ at room temperature. Nuclear staining with 4',6-diamidino-2-phenylindole-dihydrochloride (DAPI; MERCK) was performed. The primary antibodies used were rabbit anti-cfos (1:500; Santa Cruz Biotechnology catalog \#sc-52), goat anti-DCX (1:500 or 1:400; Santa Cruz Biotechnology catalog \#sc-8066), rat anti-GFP (1:500; Nacalai Tesque catalog \#04404-84), rabbit anti-GFP (1:500; Life Technologies catalog \#A11122), mouse anti-NeuN (1:500; Millipore catalog \#MAB377), rat anti-BrdU (1:1000; AbD Serotec catalog \#OBT0030G), and goat anti-lba1 (1:500; Abcam catalog \#ab5076). All secondary antibodies were purchased from Jackson ImmunoResearch Laboratories and used at 1:600 or 1:250 dilutions. The secondary antibodies used were donkey anti-rabbit-DyLight 488 (catalog \#711-485152), donkey anti-rat-Alexa 488 (catalog \#712-545-153), donkey anti-rat-Cy3 (catalog \#712-165-153), donkey anti-goat-DyLight 649 (catalog \#705-495-147), donkey anti-mouse-DyLight 649 (catalog \#715-495-151), and 
donkey anti-goat DyLight 549 (catalog \#705-505-147). Golgi staining was performed using FD Rapid GolgiStainTM kit (catalog \#PK401, FD NeuroTechnologies).

\section{Image analysis}

Epifluorescence imaging and cell counting were performed with Axio Scope A1 or Axio Imager M1 microscopes (Zeiss) with $5 \times, 10 \times$, and $20 \times$ objective lenses. Confocal imaging was conducted using an LSM710 confocal microscope (Zeiss) equipped with 488-, 543-, and $633-\mathrm{nm}$ laser lines and ZEN image-acquisition software. ImageJ software (National Institutes of Health) was used to count cells in confocal images and measure the area of granule cell layers defined by DAPI staining. The volume of the granule cell layer for individual sections was calculated by multiplying the area of the granule cell layer by the thickness of the section $(40 \mu \mathrm{m})$. Cell density was calculated by dividing the cell number by the volume of the granule cell layer. The normalized cell density indicated in Figures 2, 3 was calculated as the density in the ablated hemisphere divided by the density in the contralateral non-injected hemisphere of each mouse.

The proportion of GFP+/DCX-cells in the total granule cell population was estimated using previously reported estimates of granule cell density in the granule cell layer $\left(3.3 \times 10^{6} \mathrm{cell} / \mathrm{s} / \mathrm{mm}^{3}\right.$; Abusaad et al., 1999). The number of GFP+/DCX- cells and the volume of the granule cell layer were measured from confocal images covering the entire granule cell layer of three sections per mouse. These sections were selected from comparable rostrocaudal levels between mice.

For Figure 2E, we estimated the age of ablated new neurons as follows. Difference in the number of new neurons from the control and injected hemisphere reflects the number of ablated new neurons. We estimated the number of ablated new neurons at different ages using the following three extrapolations. (1) We used the value on day 8 to estimate the number on days 1-7. (2) The numbers between examined time points were estimated by the trapezoidal rule. (3) We assumed that new neurons up to $51 \mathrm{~d}$ old were ablated. This is because, with the trapezoid rule, the density in the control hemisphere becomes lower than that in the injected hemisphere from day 52 onwards, and the numbers of ablated new neurons become negative. Using these assumptions, the number of ablated neurons was estimated by calculating the area in the graph which is below the line formed by control hemisphere data and above the line formed by the injected hemisphere (Fig. 2D, gray area).

Confocal z-stacks for Golgi-stained granule cells were acquired, and the dendrites of Golgi-stained granule cells were traced using Neuromantic software (Myatt et al., 2012). Sholl analysis was performed in ImageJ software with Fiji package (Schindelin et al., 2012). Dendritic spines were counted under Axio Scope A1 microscope with a $100 \times$ objective lens (Zeiss).

\section{Water maze task}

Training in a series of water maze tasks was performed in a $1-m$ diameter pool placed in a dimly lit room. Dark blue curtains displaying two visual cues covered the area around the pool. The pool was filled with $17-21^{\circ} \mathrm{C}$ water $\sim 30 \mathrm{~cm}$ deep. The water was made opaque with white, non-toxic paint. The mice were moved to the water maze room in Plexiglas cages and allowed to habituate to the room for $5-10 \mathrm{~min}$ before the beginning of the experiments. For all trials except the probe trials, the mice were placed in the pool facing the wall and left to swim until they located a transparent Plexiglas circular platform $(11 \mathrm{~cm}$ in diameter). The mice were removed from the pool after $30 \mathrm{~s}$ on the platform for the first trial of the day or $15 \mathrm{~s}$ in subsequent trials. When the mice failed to locate the platform within $60 \mathrm{~s}$, they were directed toward the platform by the experimenter using a finger to point at the platform's position, and the experimenter waited for the mice to climb onto the platform.

"Pretraining" using a visible platform was performed over $4 \mathrm{~d}$ before surgery. The platform was made visible to the mice by adjusting the water level $1-2 \mathrm{~cm}$ below the platform. At the beginning of each day, the platform was moved to a new position $10 \mathrm{~cm}$ from the wall and distributed in four quadrants (NW, NE, SW, and SE). The mice underwent three trials per day. The starting positions were pseudorandomly determined from the three locations at $90^{\circ}, 180^{\circ}$, and $270^{\circ}$ from the platform.

One to $3 \mathrm{~d}$ after the completion of pretraining, viral vector injections into both hemispheres were performed. After $7 \mathrm{~d}$ of recovery, "training" using a hidden platform was performed in $1 \mathrm{~d}$. The platform was submerged $1 \mathrm{~cm}$ below the water surface. The starting positions were pseudorandomly determined from the four locations at $45^{\circ}, 135^{\circ}, 225^{\circ}$, and $315^{\circ}$ from the platform. Four blocks of three trials were performed in both the morning and the afternoon (a total of 24 trials), with a 6-h interval between the morning and afternoon sessions. The platform position was identical throughout the 24 trials. Between 5 and $10 \mathrm{~min}$ from the end of the last training trial, the mice were injected intraperitoneally with either DT $(50 \mu \mathrm{g} / \mathrm{kg})$ or an equal volume of vehicle (DPBS).

After $7 \mathrm{~d}$, spatial memory for the platform position used during training was tested in probe trials. The platform was removed from the pool, and the mice in small, clean Plexiglas cages were moved individually to the water maze room before their trials. After being transported to the water maze room, the mice were left alone for $3 \mathrm{~min}$. The mice were then placed into the pool facing the wall and allowed to swim for $1 \mathrm{~min}$ in the pool. Three probe trials were conducted for each mouse with inter-trial intervals of $1 \mathrm{~min}$. To examine memory performance without an effect of memory extinction, data from the first trials were analyzed.

Starting $1 \mathrm{~d}$ after the probe trials, the mice underwent "re-training" with eight trials per day for three consecutive days. The platform was submerged $1 \mathrm{~cm}$ below the water surface. The platform was moved to a new position each day in one of three quadrants that had not been used during training or previous re-training days. The starting position was pseudo-randomly chosen from four locations at $45^{\circ}, 135^{\circ}, 225^{\circ}$, and $315^{\circ}$ from the platform. After the final re-training trial, the mice were perfusion fixed with $4 \%$ paraformaldehyde in $0.1 \mathrm{~m} \mathrm{~PB}$. 
All trials were recorded with a video camera placed over the pool and analyzed with the ANY-maze software (Stoelting). The first probe test of one control mouse was accidentally not recorded, and data for this mouse were removed from the probe test analysis. The chance level of time spent near the platform was calculated based on the area ratio of near-platform zone to the whole pool. For occupancy plots, the time series of position data extracted from the ANY-maze software were smoothed with a twodimensional Gaussian filter and used to calculate the time spent in each pixel of the pool using custom-made programs written in MATLAB software (The MathWorks). The platform position was slightly different between groups of animals trained separately. We made the occupancy plots by rotating the entire arena so that the angles of platform centers are always at $45^{\circ}$. Radial distance from the pool center has only a few pixels difference between animals.

\section{Fear conditioning}

Fear conditioning was performed in a conditioning chamber $(24 \mathrm{~cm}$ wide $\times 20 \mathrm{~cm}$ deep $\times 30 \mathrm{~cm}$ high, Ugo Basile). Shortly after mice were moved into the chamber, the behavior of mice started being recorded with an overhead infrared red light camera. The data were analyzed for freezing behavior using EthoVision software (Noldus), where $\geq 0.5$-s continuous inactivity was detected as a freezing episode. The onset of a freezing episode was recorded as the timing of the freezing episode. After virus injection, mice were handled for $10 \mathrm{~min} / \mathrm{d}$ for $5 \mathrm{~d}$.

The protocol of trace fear conditioning generally followed Seo et al. (2015) with modifications. Training was conducted in context $A$, where a floor and walls were made of steel rods and acrylic plates, respectively, the floor and walls were cleaned with $70 \%$ ethanol before each trial and were scented with acetic acid. Training was performed in a trace conditioning protocol, which was consisted of five pairings of a tone $(5 \mathrm{kHz}, 75 \mathrm{~dB}, 20 \mathrm{~s})$ and an electrical foot-shock $(0.4 \mathrm{~mA}, 2 \mathrm{~s})$ with a 20 -s interval. Tone delivery started at $180,370,620,900$, and 1060 $\mathrm{s}$ after the start of recording. Foot-shock delivery started at $220,410,660,940$, and $1100 \mathrm{~s}$ after the start of recording. One week after the training, the tone tests were conducted in context B, where the floor was a white plastic plate and the walls were a plastic plate with a stripe pattern and the floor and wall were cleaned with Clorox Fresh Scent before a test for each mouse. Tone delivery started at $180,280,390,510$, and $620 \mathrm{~s}$ after the start of recording without pairing with a foot-shock. Data from the first tone test were analyzed. Because of accidental cessation of the task, abnormal morphology of the dentate gyrus or failure of DT or virus injections, we removed seven mice. The total numbers of mice included in the analysis were 13 and 12 mice for the control and ablated groups, respectively.

The protocol of contextual fear conditioning generally followed Deng et al. (2009) with modifications. Training, reminder training and contextual tests for contextual fear conditioning were conducted in context A. During training, mice received an electrical foot-shock $(0.7 \mathrm{~mA}, 2 \mathrm{~s})$ $180 \mathrm{~s}$ after the start of recording. The mice stayed there for additional $120 \mathrm{~s}$ before being removed from the chamber. During reminder training conducted next day, the mice received an electrical foot-shock $(0.7 \mathrm{~mA}, 0.2 \mathrm{~s}) 180$ $\mathrm{s}$ after the start of recording. The mice stayed there for additional $120 \mathrm{~s}$ before being removed from the chamber. During contextual tests, the mice stayed in the chamber for $180 \mathrm{~s}$ after the start of recording. No foot-shock was given. Data from the first contextual test were analyzed. Because of failure of virus injections, we removed two mice. The total numbers of mice included in the analysis were 20 and 18 mice for the control and ablated groups, respectively.

\section{Experimental design and statistical analyses}

Statistical analyses were performed with SPSS software (IBM) and ESCl (https://thenewstatistics.com/ itns/esci/). For independent $t$ tests (Figs. 4C,F, 5C,F, $G, 6 C, D, I-L, 7 C-E, 8 B-E, G-K)$, we first performed "Levene's test for equality of variances" in SPSS. When $p<0.05$, a $t$ test without the assumption of unequal variances was performed. When $p>0.05$, a $t$ test with the assumption of equal variances was performed. For two-way ANOVA (Fig. 5E), group and radius were used as between-subject and within-subject factors, respectively. For repeated-measures ANOVA (Figs. 2C, 3C,E, 6E, F,M $7 H-K$ ), we first performed Mauchly's test of sphericity. When $p<0.05$ for a given parameter, the Huynh-Feldt correction was applied. When interaction is significant, preplanned simple effects tests were performed by independent-sample (Figs. $2 C, 3 C, 7 H, I, K)$, one sample (Figs. $2 C, 3 C$ ), or paired (Fig. 7K) $t$ tests. Within-subject factors are hemisphere (Fig. 2C, 3C, E), day (Fig. 6E,M), trial (Fig. 6E,M), block (Fig. 6F), and period (Fig. $7 H-K$ ). Between-subject factor is maturation stage (Fig. $2 C$ ), day (Fig. $3 C, E$ ), and group (for all). All $p$ values are from two-tailed tests. All data in text and figures are presented as the mean \pm SEM. Sample size was determined considering previous studies using similar types of experiments.

\section{Data availability}

The data that support the findings of this study are available from the corresponding author on reasonable request.

\section{Results}

\section{Characterization of virus-mediated, inducible ablation method}

To examine a postlearning role of immature neurons at the early maturational stage defined by DCX expression (Brown et al., 2003), we developed a lentiviral vector bicistronically expressing DTR and GFP under the control of the DCX promoter (Fig. 1A,B). DCX is expressed in neuronal progenitors and immature neurons in the dentate gyrus (Brown et al., 2003). In adult mice, DCX expression starts declining at approximately two weeks after neuronal birth and then decreases further along neuronal maturation (Kempermann et al., 2003). Therefore, DTR expression is expected to be limited to neuronal progenitors and immature neurons. GFP expression was localized predominantly along the hilar border of the granule cell layer across the AP axis of the dentate gyrus, where adult-born neurons are 
A

LTR DCX promoter DT receptor IRES GFP WPRE LTR

B

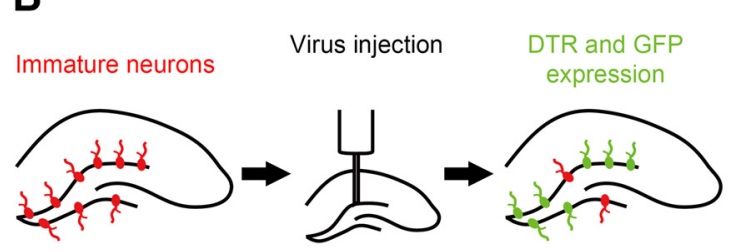

DT injection

(i.p.)

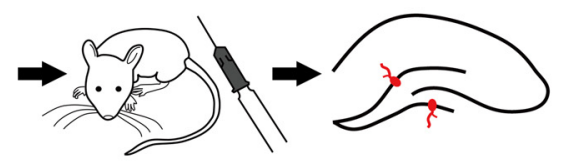

F

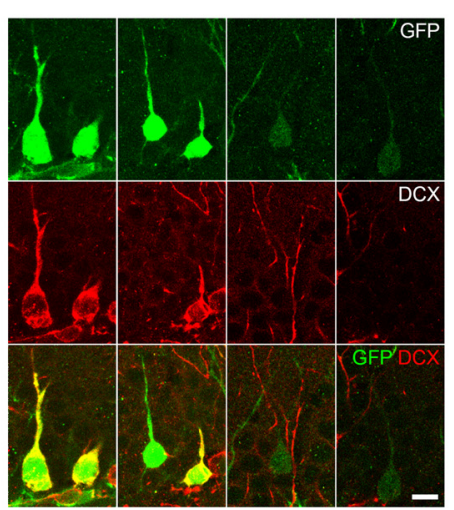

C

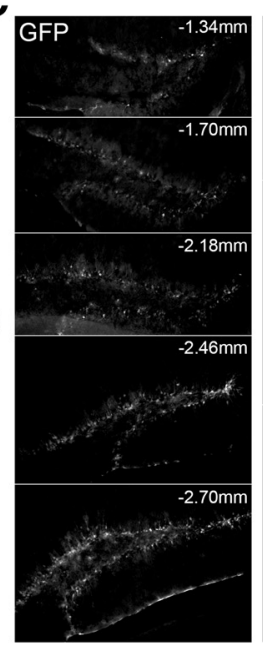

G

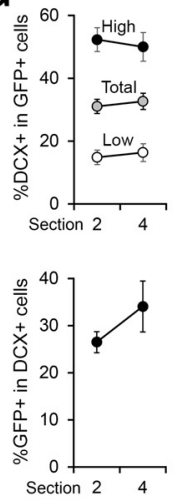

H

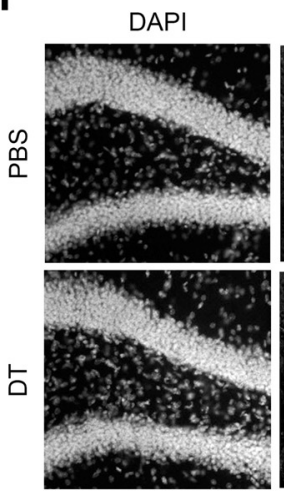

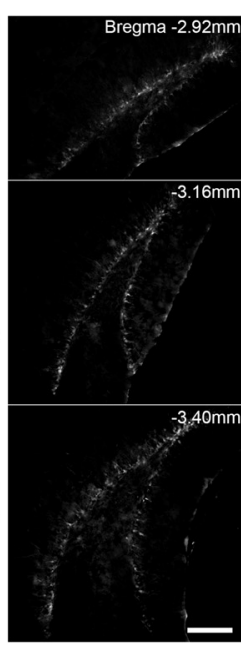

DCX
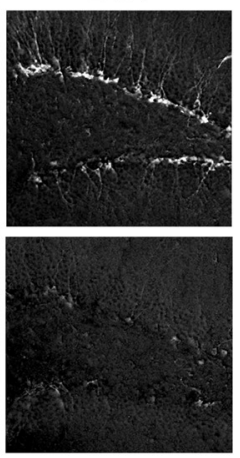

D

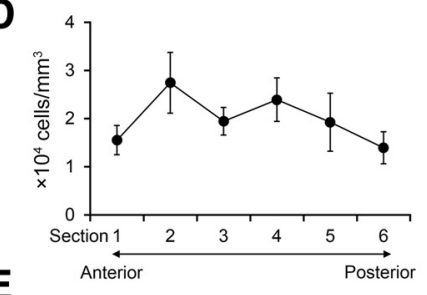

E

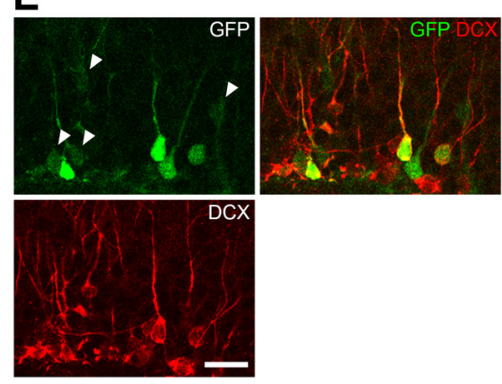

GFP
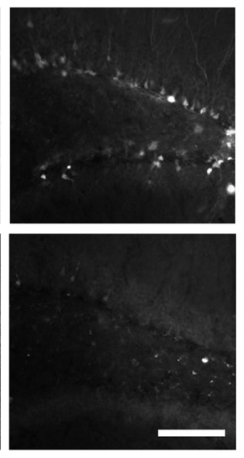

Figure 1. Diphtheria toxin-induced ablation of DCX+ cells in the dentate gyrus using lentiviral transduction of DTR. $\boldsymbol{A}$, Schematic representation of the recombinant lentiviral vector construct. LTR: long terminal repeat; DCX: doublecortin; DT: diphtheria toxin; IRES: internal ribosome entry site; GFP: green fluorescent protein; WPRE: Woodchuck hepatitis virus posttranscription regulatory element. $\boldsymbol{B}$, Diagram of the ablation technique specific for immature neurons. $\boldsymbol{C}-\boldsymbol{E}$, To evaluate the distribution and specificity of viral transduction, we injected the viral vector into the dentate gyrus of mice and examined GFP expression $7 \mathrm{~d}$ after the injection, which corresponds to the time when DT was injected in behavioral experiments (Figs. 6-8). C, Representative images showing the distribution of virus-transduced cells visualized by GFP fluorescence over the AP axis of the dentate gyrus. GFP expression was localized predominantly along the hilar border of the granule cell layer across the AP axis of the dentate gyrus, where adult-born neurons are known to be located. Scale bar: $200 \mu \mathrm{m}$. $\boldsymbol{D}$, Density of GFP + cells across the AP axis of the dentate gyrus. GFP+ cells were quantified in every 12 coronal sections covering the AP axis of dentate gyrus. Sections 1 and 6 correspond to $\sim 1.06-1.34$ and 3.52-3.80 $\mathrm{mm}$ posterior to the bregma, respectively. $\boldsymbol{E}$, A representative confocal image of the granule cell layer in a section immunostained against GFP and DCX. Among $460 \mathrm{DCX}+$ cells examined (from one hemisphere in three sections each of two mice), $40.7 \%$ of $\mathrm{DCX}+$ cells expressed GFP, indicating that viral transduction was achieved in a large proportion of immature neurons. Among 455 GFP + cells examined (from one hemisphere in three sections each of two mice), $43.8 \%$ of GFP + cells were DCX + . We noted that GFP expression level was highly variable between GFP+ cells (low GFP-expressing cells are highlighted by arrowheads). Scale bars: $20 \mu \mathrm{m}$. $\boldsymbol{F}, \boldsymbol{G}$, To further evaluate the distribution and specificity of viral transduction, we analyzed sections from nine control mice used for the behavioral experiment described in Figure 6. These sections were from $17 \mathrm{~d}$ after virus injection, during which water maze training, probe trials and re-training were performed. $\boldsymbol{F}$, Representative confocal images showing colocalization of DCX and GFP in neurons in the granule cell layer. Scale bars: $10 \mu \mathrm{m}$. G, Top, Proportion of GFP+ cells expressing DCX. Percentage within total population, high and low GFP-expressing cell populations were plotted separately. Bottom, Proportion of DCX + cells expressing GFP. For each population, two sections from different AP levels corresponding to sections 2 and 4 in $\boldsymbol{D}$ were analyzed separately. We observed some GFP+DCX- cells which appeared to include non-neuronal and granule cells based on their morphology. Without an active degradation mechanism, GFP protein is highly stable even after the transcription activity of the promoter is shut off (Andersen et al., 1998). Therefore, GFP protein is expected to be maintained in new neurons that expressed DCX at the time of virus injection but thereafter lost the expression of DCX (and presumably DTR) during the survival time. Thus, some of GFP+DCX-cells were likely to be new neurons that had just lost DCX expression. In accordance with this possibility, the proportion expressing DCX was higher in high GFP-expressing cells (>50\%) than that of low-expressing cells $(<20 \%)$. Nonetheless, we cannot completely exclude the possibility that some of GFP + cells were mature neurons. Our estimation revealed that the proportion of GFP+/DCX-cells among the total granule cell population in the dentate gyrus would be small (0.6\%; see Materials and Methods). This is consistent with our observation of no ablation of mature granule cells (nine weeks old), shown in Figure 2. $\boldsymbol{H}$, Fluorescent images of the virus-injected dentate gyrus after the injection of PBS or DT. 
continued

Top, The granule cell layer visualized by DAPI staining was intact after DT-induced ablation. Middle and bottom, DT injection led to an obvious reduction in DCX + and GFP + cells. Scale bar: $100 \mu \mathrm{m}$.

located (Fig. 1C,D). Immunostaining for DCX revealed that up to $40.7 \%$ of DCX + cells expressed GFP, indicating that viral transduction was achieved in a large proportion of immature neurons (Fig. 1E-G). As a proof of principle for the ablation method, we injected the viral vector into the dentate gyrus, and one week later, we intraperitoneally injected DT for ablation or PBS as a control. After an additional oneweek survival period, we perfused the mice and examined $\mathrm{DCX}+$ cells in the dentate gyrus. We observed a clear reduction in the density of DCX + cells in DT-injected mice compared with that in PBS-injected controls (Fig. $1 \mathrm{H}$; quantitative results of larger cohorts in Fig. $6 C, 7 D, 8 B$ ).

Next, to determine the maturational stage of ablated neurons, we labeled different ages of adult-born neurons by injecting a thymidine analog, BrdU, into three groups of mice 8-14 d (for DCX + immature neurons), four weeks (for young neurons mostly DCX-negative) and nine weeks (for mature neurons) before DT injection (Fig. 2A). One week before the DT injection, the viral vector was injected into the dentate gyrus of one hemisphere of all mice. We perfused the mice one week after the DT injection and analyzed the density of adultborn neurons (BrdU $+/ \mathrm{NeuN}+$ cells; Fig. 2B,C). The normalized density and age of adult-born neurons showed a significant, positive correlation $(r=0.802$, $p=9.7 \times 10^{-4}, n=13$ mice), indicating that younger neurons were more selectively eliminated by the ablation method. Further, the normalized densities of 8- to 14-d-old neurons were significantly reduced in the granule cell layer of the virus-injected hemisphere compared with the control hemisphere $[65.8 \pm 5.7 \%$ reduction; two-way (hemisphere $\times$ maturation stage) repeated measures ANOVA, hemisphere: $p=5.9 \times 10^{-4}, F_{(1,10)}=24.368$, maturation stage: $p=2.2 \times 10^{-4}, F_{(2,10)}=22.059$, hemisphere $\times$ maturation stage: $p=2.2 \times 10^{-4}, F_{(2,10)}=22.059$, $n=13$ mice in total; $p=3.3 \times 10^{-4}, t_{(4)}=-11.440, n=5$ mice, one sample $t$ tests against 1]. On the other hand, the normalized density of four- and nine-week-old neurons did not show a significant reduction (four weeks: $p=0.449$, $t_{(3)}=-0.869$; nine weeks: $p=0.474, t_{(3)}=0.816, n=4$ mice for each, one sample $t$ tests against 1$)$. The normalized densities of 8- to 14-d-old neurons were significantly lower than four-week-old neurons $\left(p=0.0028, t_{(7)}=-4.502\right.$, independent-sample $t$ test] and nine-week-old neurons $\left(p=8.7 \times 10^{-5}, t_{(7)}=-8.057\right)$. It is known that DCX expression occurs in $>80 \%$ of new neurons at less than two weeks of neuronal age, reduces to a large extent at four weeks and is then virtually none at neuronal maturity (Jagasia et al., 2009; Snyder et al., 2009). Thus, the pattern of ablation is consistent with DCX expression along neuronal maturation. Furthermore, 77.4 and $90.2 \%$ of the ablated new neurons are estimated to be younger than 14 and $21 \mathrm{~d}$ old at the time of DT injection, respectively (Fig. $2 D, E$; for details in estimation, see Materials and Methods), indicating that most ablated neurons were at the early maturational stage.
Next, to characterize the time course of neuronal ablation, we injected the viral vector into one hemisphere of the dentate gyrus and administered DT one week later (Fig. 3A). Either 1, 4, or $7 \mathrm{~d}$ after the DT injection, we analyzed DCX + cell density. The density in the injected hemispheres were normalized by dividing by the density in noninjected hemispheres in the same mice. Normalized DCX+ cell density showed a significant, negative correlation with days after DT injection $\left(r=-0.843, p=5.7 \times 10^{-4}, n=12\right.$ mice; Fig. $3 B, C$ ), indicating that normalized $D C X+$ cell density reduced over time. Further we performed two-way (hemisphere $\times$ day) repeated measures ANOVA, and detected significance in the main effect of day and hemisphere and hemisphere $\times$ day interaction (day: $p=0.0023$, $F_{(2,9)}=12.869$, hemisphere: $p=2.0 \times 10^{-5}, F_{(1,9)}=65.419$, hemisphere $\times$ day: $p=0.0023, F_{(2,9)}=12.869, n=12$ mice in total). One sample $t$ test against 1 indicated that the normalized density of DCX + cells were significantly reduced in injected hemispheres than in non-injected controls at all three time points $\left(1 \mathrm{~d}: p=3.0 \times 10^{-4}, t_{(3)}=-19.484,4 \mathrm{~d}\right.$ : $\left.p=0.016, t_{(3)}=-4.906,7 \mathrm{~d}: p=0.011, t_{(3)}=-5.573\right)$. Independent sample $t$ test between days showed that the normalized density at $4 \mathrm{~d}$ after DT injection was not significantly lower than that at $1 \mathrm{~d}\left(p=0.062, t_{(3.064)}=2.880\right.$, independent $t$ test), but reached significance at $7 d(p=0.020$, $\left.t_{(3.016)}=4.548\right)$. Thus, the ablation of immature neurons gradually occurred over multiple days.

Most ablation methods used in similar studies induces inflammation-like response (Arruda-Carvalho et al., 2011; Seo et al., 2015). Therefore, we quantified the number of lba1 + cells in the dentate gyrus, whose increase indicates inflammation-like response. An increase in the number of lba1 + cells was detected after the induction of ablation by a combination of viral vector and DT injections (Fig. 4). It is known that inflammation can cause a detrimental effect on brain functions including memory, which is associated with damages in neuronal structures (DiSabato et al., 2016). We found that the volume (Figs. $1 H, 5 A, B$; quantitative results of larger cohorts in Fig. $6 D, 7 E, 8 C$ ) and cell density (Fig. $5 B, C$ ) of the granule cell layer remained intact. The morphology of mature granule cells (Fig. 5D-G) was also unaffected. These observations indicate that DT injection ablated $\mathrm{DCX}+$ cells without inducing the large-scale elimination or damage of mature neurons in the granule cell layer. We found that the ablation technique does not affect neurogenesis either in the olfactory bulb or in the subventricular zone (Fig. $5 \mathrm{H}$ ).

In the experiment in which we examined the time course of ablation, we also assessed the potential non-specific effects of ablation on the activity of other neurons in the granule cell layer. We analyzed the expression of an immediate early gene product, c-fos protein, as a proxy for protein synthesis and neuronal activation (Fig. 3D,E). To induce c-fos expression, the mice were exposed to a novel environment $90 \mathrm{~min}$ before perfusion. Two-way 
A

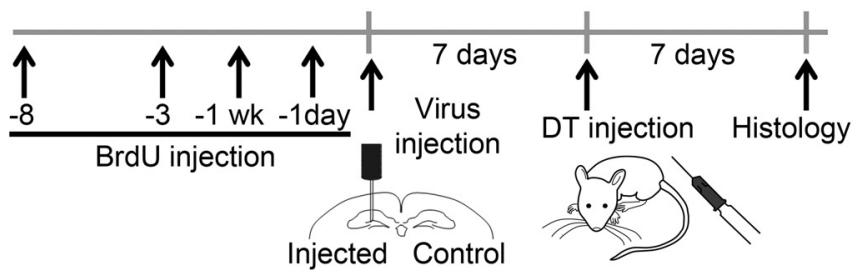

B

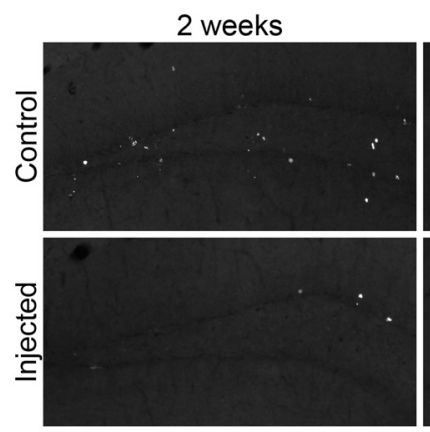

$E$

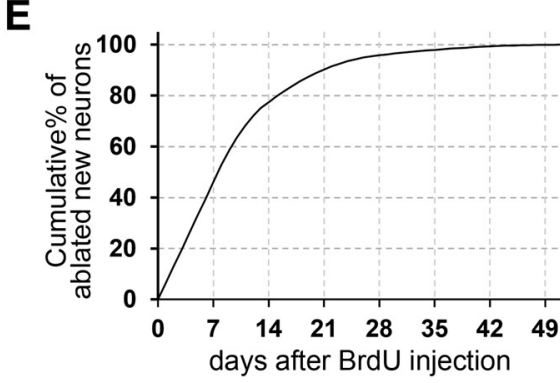

Figure 2. DT-induced ablation of new neurons at the early maturational stage. A, Experimental design. To label the different ages of newborn neurons, BrdU was injected into mice at four different time points ( $8 \mathrm{~d}, 14 \mathrm{~d}, 4$ weeks, and 9 weeks) before DT administration. For each mouse, one dose of BrdU (100 $\mu \mathrm{g} / \mathrm{g}$ body weight) was injected intraperitoneally. B, Representative images showing two- or nine-week-old BrdU+ cells from virus-injected and non-injected control hemispheres after DT injection. Scale bar: $150 \mu \mathrm{m}$. $\boldsymbol{C}$, Normalized densities of BrdU+/NeuN + cells. The densities in the injected hemispheres were normalized by dividing by the densities in the non-injected hemispheres of the same mice. Each data point represents one mouse ( $8 \mathrm{~d}$ : $n=3$ mice, two weeks: $n=2$, four weeks: $n=4$, nine weeks: $n=4)$. A value of 1 indicates that the densities of both hemispheres are equal. Note that a significant reduction in immature (8- to 14-d-old) neurons but minimal effects on four- and nine-week-old neurons. The densities were measured from three sections (every $1240-\mu \mathrm{m}$-thick sections, corresponding to sections $2-4$ in Fig. $1 D$ ) for each mouse. These three sections are distributed over the dorsal region of the dentate gyrus. $\boldsymbol{D}$, Estimation of the numbers of ablated neurons at different ages, using trapezoidal rule integration. Difference in the number of new neurons between the control and injected hemisphere (gray area) reflects the number of ablated new neurons. $\boldsymbol{E}$, Cumulative percentage of ablated new neurons at different ages, using trapezoidal rule integration. Percentages of ablated neurons younger than different ages in the total ablated population were plotted; ${ }^{* *} p<0.005$.

(hemisphere $\times$ day) repeated measures ANOVA did not detect any significance in the main effects or interaction (day: $p=0.613, F_{(2,9)}=0.516$, hemisphere: $p=0.772, F_{(1,9)}=0.090$, hemisphere $\times$ day: $\left.p=0.613, F_{(2,9)}=0.516\right)$. While sample number is limited, c-fos protein expression was similar between injected and control hemispheres (95\% confidence interval of difference in hemisphere effect, [-0.011, 0.066]; in day $1,[-0.251,0.131]$; in day $4,[-0.138,0.210]$; in day 7 , $[-0.253,0.225])$. In addition, we did not detect significant correlation between the normalized c-fos + cell density and days after DT injection $(r=0.144, p=0.654,95 \%$ confidence interval of $r,[-0.469,0.663])$. Thus, using limited number of samples, we did not detect any clear structural and functional sign of damages which inflammation may have caused.

\section{Postlearning ablation impaired the persistent search for missing platform in probe tests of a water maze task}

Next, we induced the ablation after task training in a water maze task and examined its effects on their performance in a probe test (Fig. 6A). First, we pretrained the mice over $4 \mathrm{~d}$ using a visible platform to familiarize them with the water maze procedures (pretraining). The mice were bilaterally injected in the dentate gyrus with the viral vector. After a 7-d recovery period, the mice were trained in a hippocampus-dependent water maze task using a hidden platform in a constant position over 24 trials in $1 \mathrm{~d}$ (training; Trouche et al., 2009). At the completion of the 24 trials, DT was systemically administered to the mice to induce the ablation (ablated group). Seven days after training, probe tests were performed to evaluate memory for the position where the platform was located during training. These tests were followed by re-training with new platform positions each day over $3 \mathrm{~d}$. The control group were trained and tested in the same protocol except ablation was not induced; a half of control group was injected with vehicle instead of the viral vector and the other half was injected with vehicle instead of DT. We confirmed a reduction of DCX + cells in the ablated group $(p=0.011$, $t_{(31.241)}=2.688, n=18$ mice for each group, independent sample $t$ test; Fig. $6 B, C)$, while the volume of the granule cell layer was intact $\left(p=0.939, t_{(34)}=0.077\right.$, independent sample $t$ test; Fig. $6 B, D$ ). In pretraining and training (which were conducted before the ablation was induced), both groups learned the platform positions similarly well (Fig. $6 E, F$; Table 1). 
A

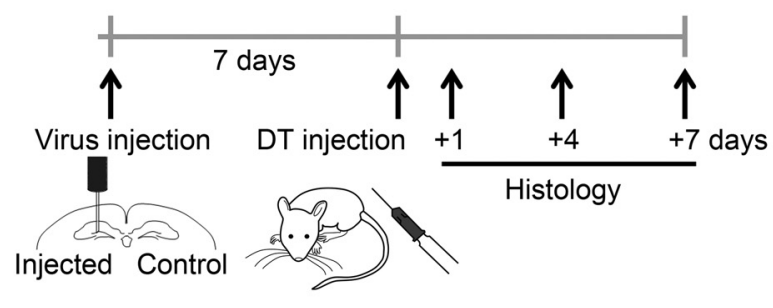

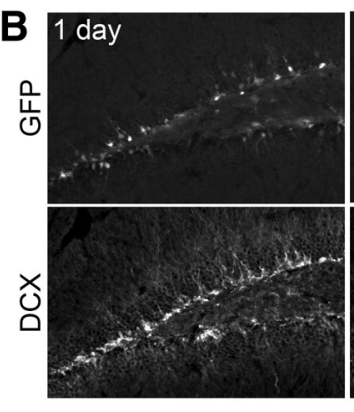
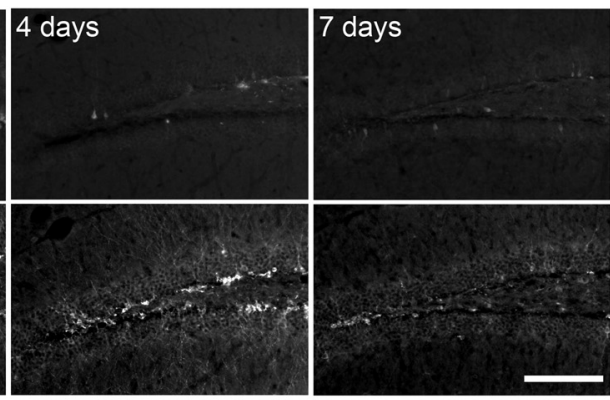

E

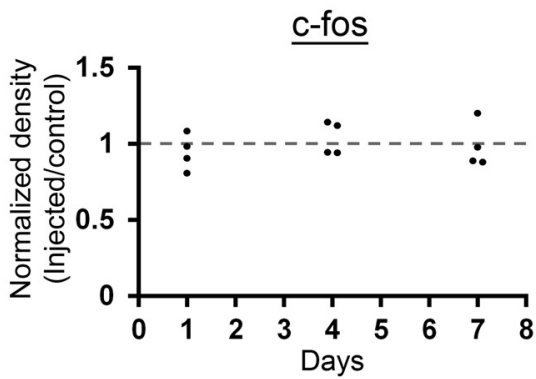

Figure 3. Ablation of DCX+ cells occurs over multiple days while keeping the ability of protein synthesis/activity-dependent gene expression intact in the granule cell layer. $\boldsymbol{A}$, Experimental design. $\boldsymbol{B}$, Representative images showing the reduction of GFP+ cells over $7 \mathrm{~d}$ after DT injection. Scale bars: $150 \mu \mathrm{m}$. C, Normalized densities of DCX+ cells in the virus-injected hemisphere after DT injection. The densities in the injected hemispheres were normalized by dividing by the densities in non-injected hemispheres in the same mice. $\boldsymbol{D}$, Representative images of $\mathrm{c}$-fos expression in the granule cell layer of virus-injected and non-injected control hemispheres. Scale bars: $100 \mu \mathrm{m}$. $\boldsymbol{E}$, Normalized densities of c-fos + cells over $7 \mathrm{~d}$ after DT injection. The same way of normalization as $\boldsymbol{C}$ was used.

For the probe tests, we analyzed (1) the time that the mice spent in proximity to the platform and (2) the number of entries into the area where the platform was located (Fig. 6G,H). The ablated group ( $n=18$ mice) showed significantly lower values in both parameters than the control group $\left(n=17\right.$ mice; $p=0.008, t_{(27.035)}=2.877$ and $p=0.019$, $t_{(33)}=2.465$, respectively, independent-sample $t$ tests; Fig. $61)$, whereas both control and ablated groups spent significantly longer time near platform than the chance level (4.3 s; control: $p=2 \times 10^{-6}, t_{(16)}=7.244$, ablated: $p=1.1 \times 10^{-5}$, $t_{(17)}=6.151$, one-sample $t$ test; Fig. 6/). These results indicate that, while both groups remembered the platform position, the ablated group showed a deficit in memory retrieval.

To further examine the details in pattern of the deficit, we quantified other parameters. Unexpectedly, latency to reach former platform area was comparable between the two groups $\left(p=0.922, t_{(33)}=-0.099\right.$, independent-sample $t$ test; Fig. 6J), suggesting that performance impairment occurred specifically after the mice reached the former platform area. This is why we focused on the period after the first entry to the platform position. Similar to the whole-duration analysis in Figure 6/, the proportion of time in which the mice spent near platform and the number of entries into the former platform area per unit time were significantly lower in the ablated group than the control group $\left(p=0.0062, t_{(23.352)}=3.008\right.$ and $p=0.013$, $t_{(33)}=2.615$, respectively, independent-sample $t$ tests; Fig. 6K). These results indicate that memory retrieval was initially intact in the ablated group and as accurate as the control group; after initial failure to find the platform the control group persisted to search platform while the ablated group reduced search prematurely. In addition, we found that the duration of individual visits near platform was shorter in the ablated group $\left(p=0.022, t_{(20.741)}=\right.$ 2.469, independent-sample $t$ test; Fig. $6 L$ ). Although the time scale is different, this result also indicates that persistence of platform search was impaired in the ablated group. Swimming speed was not significantly different between the groups $\left(p=0.884, t_{(33)}=0.148\right.$, independentsample $t$ test; Fig. 6/). In re-training, both groups learned the platform positions similarly well (Fig. 6M; Table 1).

\section{Postlearning ablation impaired the persistence of tone-induced freezing behavior after tone trace fear conditioning}

To test whether the effect of the postlearning ablation on persistence of learned behavior is generalized to another form of hippocampus-dependent memory, we examined the effect of the postlearning ablation on a trace fear conditioning task. Similarly, to the water maze experiment, one week after virus injection, mice received training for trace fear conditioning (Fig. 7A). The mice were exposed to five pairings of a tone (a conditioned stimulus) and an electrical shock (an unconditioned stimulus) with a 20-s interval in context A (Fig. 7B). After the completion of training, mice in the control and ablated groups $(n=13$ and 12 mice, respectively) received systemic injection of PBS and DT, respectively. The two groups showed similar freezing level during training (which was conducted before inducing the ablation) both before and after the first tone was given $\left(p=0.471, t_{(23)}=0.733\right.$ and $p=0.643$, $t_{(23)}=-0.470$, respectively, independent-sample $t$ tests; 
A
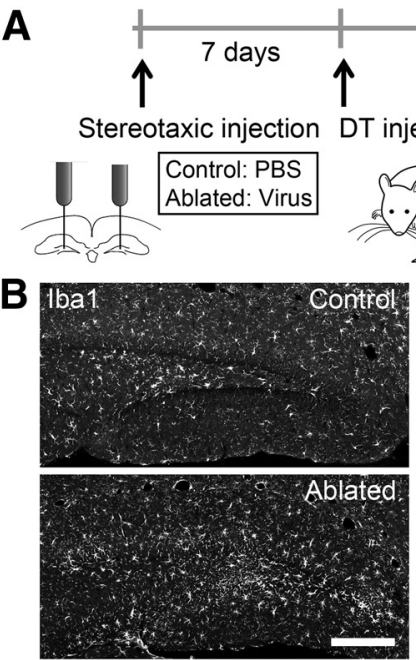

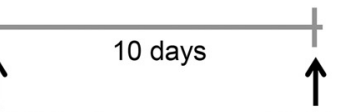

DT injection

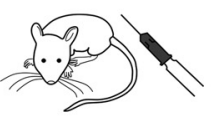

C

C Iba1+ cell density

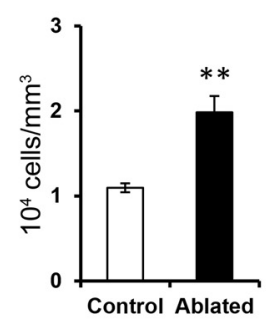

D

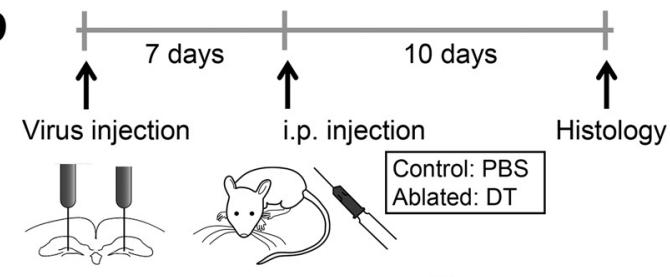

E

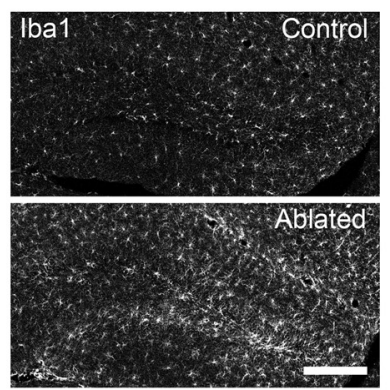

F $\underline{\text { lba } 1+\text { cell density }}$

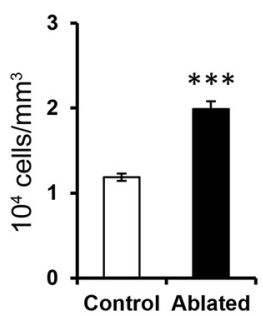

Figure 4. Ablation method induces Iba1 expression in the dentate gyrus. $\boldsymbol{A}$, Experimental design. Mice were stereotaxically injected with PBS (control group) or the viral vector (ablated group) into the dentate gyrus. After $7 \mathrm{~d}$ of recovery, DT was injected into both groups. Brain sections were prepared from the mice after an additional $10 \mathrm{~d}$ of survival. $\boldsymbol{B}$, Representative images of lba1 immunostaining of brain sections from the control and ablated groups. $\mathbf{C}$. Density of Iba1+ cells in the dentate gyrus. An increase was observed after the induction of ablation by a combination of the viral vector and DT injections $\left(p=0.0081, t_{(4.566)}=-4.478, n=5\right.$ for each group, independent sample $t$ test). $\boldsymbol{D}$, Experimental design. Mice were stereotaxically injected with the viral vector into the dentate gyrus. After $7 \mathrm{~d}$ of recovery, PBS (control group) or DT (ablated group) was injected into both groups. Brain sections were prepared from the mice after an additional $10 \mathrm{~d}$ of survival. $\boldsymbol{E}$, Representative images of lba1 immunostaining of brain sections from the control and ablated groups. $\boldsymbol{F}$, Density of Iba1 + cells in the dentate gyrus. An increase was observed after the induction of ablation by a combination of the viral vector and DT injections $\left(p=3.9 \times 10^{-5}, t_{(8)}=-8.132, n=5\right.$ for each group, independent sample $t$ test).

Fig. 7C). We confirmed a reduction of DCX + cells in the ablated group $\left(p=3 \times 10^{-6}, t_{(15.705)}=7.096\right.$, independent sample $t$ test; Fig. 7D) while the volume of the granule cell layer was intact $\left(p=0.556, t_{(23)}=0.597\right.$, independent sample $t$ test; Fig. 7E).

One week after training, mice underwent a tone test in context $\mathrm{B}$, in which mice were exposed to five tones without a foot shock (Fig. 7F,G). We analyzed percentage time in freezing during $180 \mathrm{~s}$ baseline period (before first tone exposure) and five consecutive 20 -s periods (tone, posttones 1, 2, 3, and 4) averaged over five tone exposures (Fig. 7F,H). Two-way (period $\times$ group) repeated measures ANOVA detected significant effects of period and group and significant interaction between period and group (period: $p=2.0 \times 10^{-22}, F_{(4.242,115)}=46.378$, group: $p=0.021$, $F_{(1,23)}=6.117$, period $\times$ group: $p=0.002, F_{(4.242,115)}=4.576$; Fig. $7 H$ ). The two groups showed comparable level of freezing during the baseline period and similarly increased freezing level in Tone period $\left(p=0.252, t_{(23)}=1.175\right.$, and $p=0.926, t_{(23)}=0.094$, respectively, independent-sample $t$ tests). However, from posttone 1 onwards, freezing level of the ablated group became lower than the control group, which became significant during posttone 3 and 4 periods (posttone 1: $p=0.055, t_{(23)}=2.021$, posttone 2: $p=0.081$, $t_{(23)}=1.828$, posttone $3: p=0.001, t_{(20.222)}=3.755$, post tone 4: $p=0.017, t_{(18.333)}=2.618$, independent-sample $t$ tests). When we focused on the response to the first tone exposure only (instead of average over 5 exposures), the ablated group showed a similar reduction specifically at later time points (period: $p=5.6 \times 10^{-14}, F_{(5,115)}=19.419$, group: $p=0.064, F_{(1,23)}=3.786$, period $\times$ group: $p=4.5 \times$ $10^{-5}, F_{(5,115)}=6.128$, two-way repeated measures ANOVA; baseline: $0.252, t_{(23)}=1.175$, tone: $p=0.404, t_{(23)}=-0.851$, posttone $1,2,3,4: p=0.522,0.139,8.4 \times 10^{-4}, 0.014$, $t_{(23)}=0.651,1.532,3.839,2.662$, respectively, independent-sample $t$ tests; Fig. 7I). Thus, freezing response of the ablated group was initially intact and increased similarly to the control group. However, this increase did not persist as long as it did in the control group.

We have also analyzed the frequency and duration of individual freezing episodes. The frequency of freezing episodes did not show a significant difference between the two groups and similarly increased from baseline to after the first tone exposure (period: $p=3.2 \times 10^{-7}, F_{(1,23)}=50.200$, group: $p=0.743, F_{(1,23)}=0.110$, period $\times$ group: $p=0.097$, $F_{(1,23)}=2.990$, two-way repeated measures ANOVA; Fig. 7 ). For the duration of freezing episodes (Fig. 7K), two-way repeated measures ANOVA (period $\times$ group, control: $n=13$ mice, ablated: $n=12$ ) detected a significant effect of period $\left(p=4.5 \times 10^{-8}, F_{(1,23)}=63.568\right)$ and group $\left(p=0.029, F_{(1,23)}=\right.$ 5.427) and significant interaction (period $\times$ group: $p=0.017$, $\left.F_{(1,23)}=6.571\right)$. In the baseline period, the duration was comparable between the groups $\left(p=0.356, t_{(23)}=0.942\right.$, independent-sample $t$ test). In contrast, after the onset of first tone, the duration was significantly shorter in the ablated group than in the control group $\left(p=0.019, t_{(16.486)}=2.600\right.$, independent-sample $t$ test). Thus, the persistence of individual freezing episodes was impaired by the postlearning ablation.

\section{Postlearning ablation impaired the persistence of context-induced freezing behavior after contextual fear conditioning}

Next, we examined whether the postlearning ablation affects contextual fear conditioning, another form of 
A

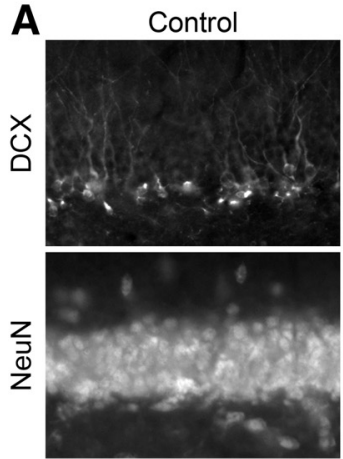

D

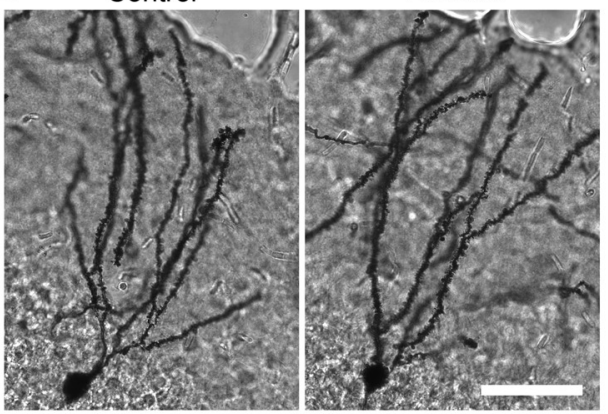

H

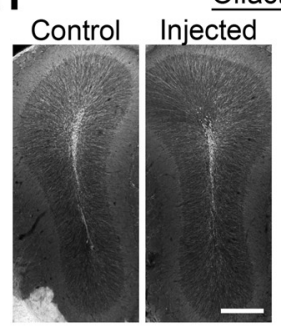

Injected

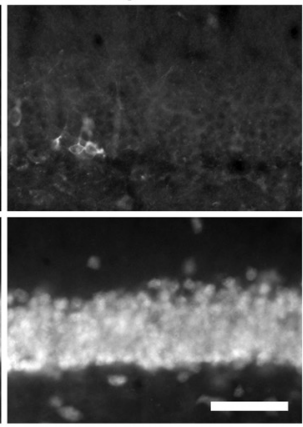

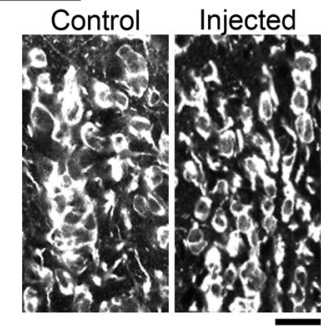

B

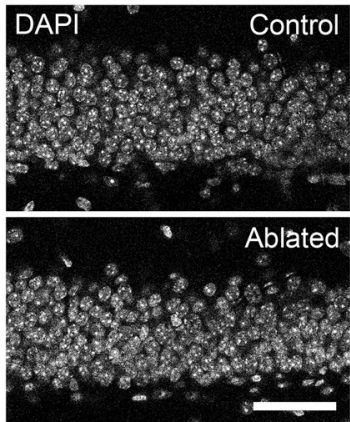

C

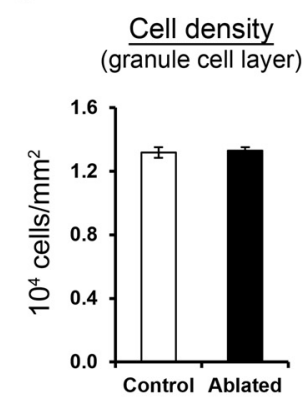

$\mathbf{F}$
G Spine density
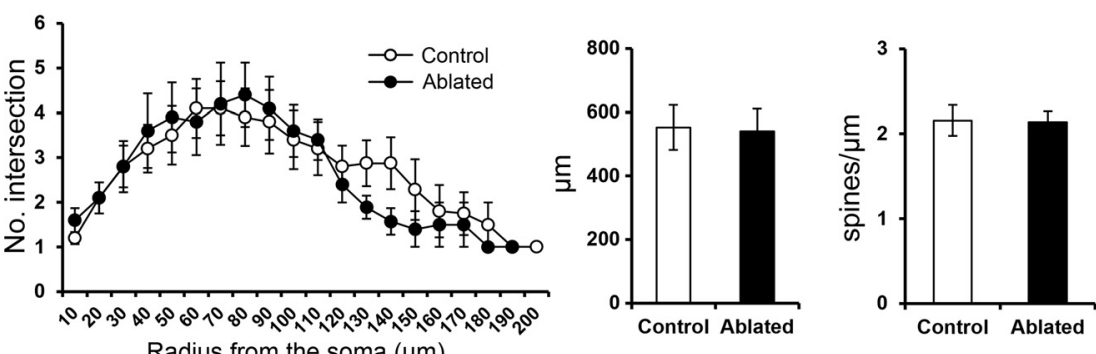

Radius from the soma $(\mu \mathrm{m})$

Subventricular zone
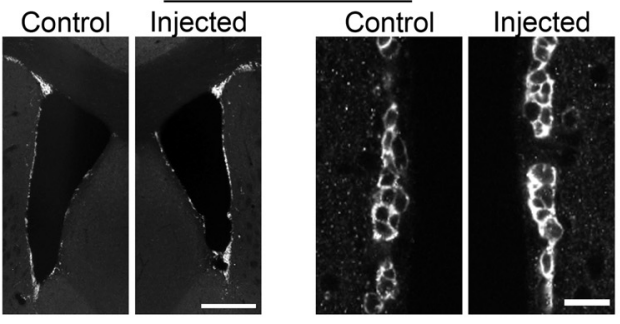

Figure 5. Overall integrity of the granule cell layer and mature granule cells is intact after DT-induced ablation. $\boldsymbol{A}$, NeuN immunostaining showed the integrity of granule cell layer after DT-induced ablation. The lentiviral vector was injected into the dentate gyrus in one hemisphere. Seven days later, DT was injected. After another 7-d survival, brain sections were prepared and immunostained with DCX or NeuN. The images were from virus-injected (Injected) and non-injected (control) hemispheres. Scale bar: $50 \mu \mathrm{m}$. B, DAPI immunostaining showing the integrity of granule cell layer of mice in the control and ablated group. Scale bar: $50 \mu \mathrm{m}$. $\boldsymbol{C}$, Cell density in the granule cell layer was not affected by the ablation $\left(p=0.783, t_{(23)}=-0.279\right.$, control: $n=13$ mice, ablated: $n=12$, independent sample $t$ test). The analysis was performed using the sections from the mice used in the experiments described in Figure 7 . $\boldsymbol{D}$, Golgi-stained granule cells of mice in the control and ablated group. The lentiviral vector was injected into the dentate gyrus in both hemisphere, and PBS or DT was systemically injected $7 \mathrm{~d}$ later. After another 7-d survival, the mice were euthanized for the Golgi staining. Scale bar: $50 \mu \mathrm{m}$. $\boldsymbol{E}$, Sholl analysis for Golgi-stained granule cells in the control and ablated group. No significant group difference was detected (group: $p=0.600, F_{(1,271)}=0.276$, radius: $p=1.1 \times 10^{-9}, F_{(19,271)}=4.429$, group $\times$ radius interaction: $p=0.996, F_{(18,271)}=0.330, n=10$ cells for each group, two-way ANOVA). $\boldsymbol{F}$, Total dendritic length of the Golgi-stained granule cells. No significant, group difference was detected $\left(p=0.907, t_{(18)}=0.119, n=10\right.$ cells for each group, independent sample $t$ test). $G$, The density of dendritic spines in the Golgi-stained granule cells. No significant group difference was detected $\left(p=0.923, t_{(18)}=0.098\right.$, $n=10$ cells for each group, independent sample $t$ test). $\boldsymbol{H}$, Intact olfactory bulb neurogenesis after DT-induced ablation in the dentate gyrus. Fluorescent images of DCX+ cells in the olfactory bulb and subventricular zone after DT-induced ablation in the dentate gyrus. Images from virus-injected and control hemispheres are shown. To confirm that the ablation technique did not affect neurogenesis outside the dentate gyrus, we injected the viral vector into the dentate gyrus in one hemisphere and performed DT injection one week after the virus injection. We examined the densities of DCX + cells one week after the DT injection and did not observe any obvious difference between the virus-injected hemisphere and non-injected control hemisphere either in the olfactory bulb or in the subventricular zone. This observation was confirmed by quantifying the density of DCX + cells in the subventricular zone (control hemisphere: $2.97 \pm 0.02$; ablated hemisphere: $3.22 \pm 0.15$ in $10^{3}$ cells $/ \mathrm{mm}^{2}, p=0.248, t_{(2)}=-1.614, n=3$ mice, paired $t$ test) and the granule cell layer of the olfactory bulb (control hemisphere: $5.35 \pm 1.47$; ablated hemisphere: $5.68 \pm 1.14$ in $10^{4} \mathrm{cells} / \mathrm{mm}^{3}$, $p=0.825, t_{(2)}=-0.252, n=3$ mice, paired $t$ test). Scale bars: $300 \mu \mathrm{m}$ (left) and $15 \mu \mathrm{m}$ (right); ${ }^{\star \star} p<0.01,{ }^{\star \star *} p<0.005$. Scale bars: $300 \mu \mathrm{m}$. 
A

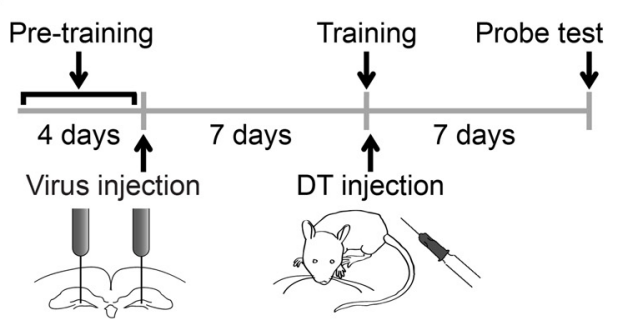

C

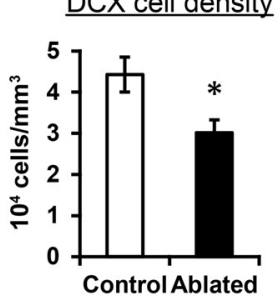

D

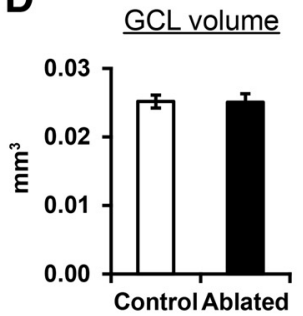

B

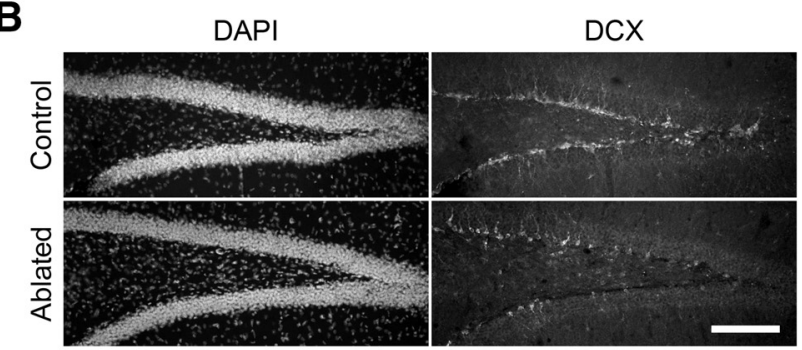

E

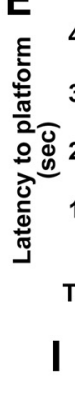

Pre-training

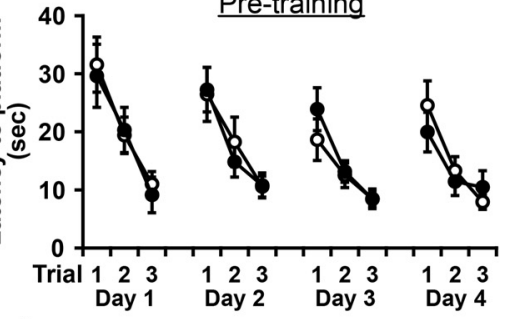

Entire trial
F

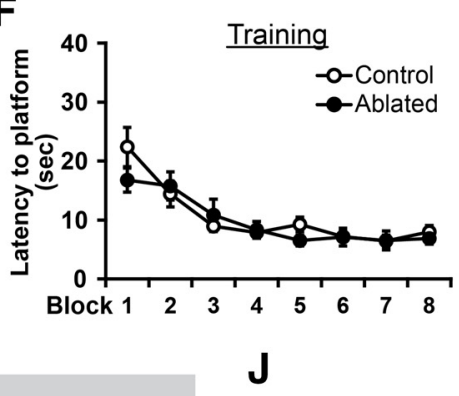

$J$
G

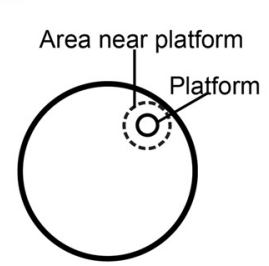

H

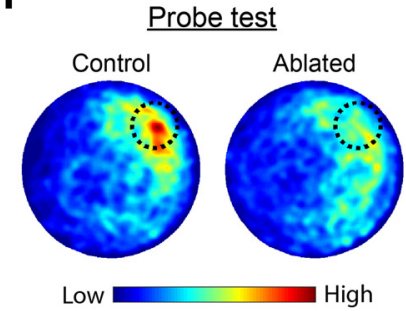

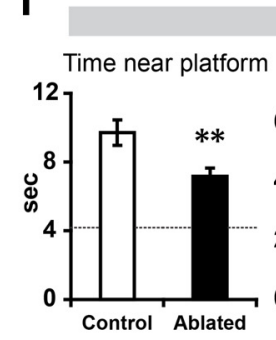
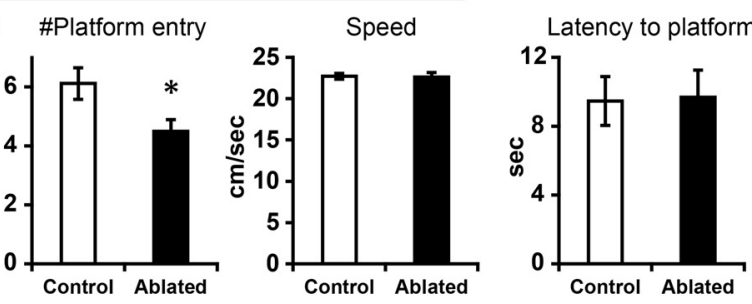

K

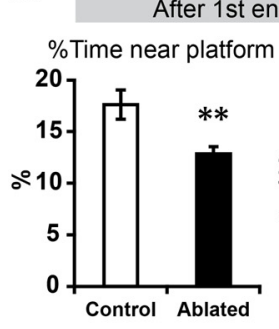

$\mathbf{L}$

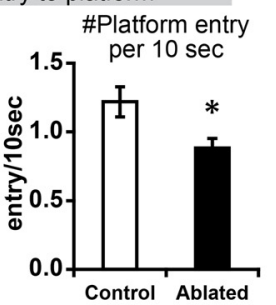

E Entire trial Duration per visit
M

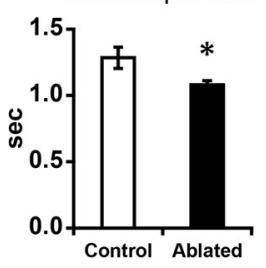

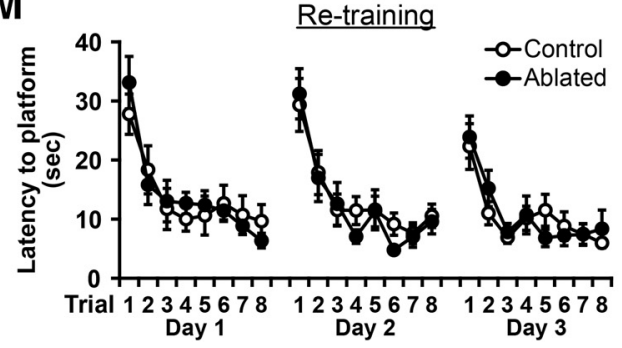

Figure 6. Posttraining ablation impaired the persistence of platform search behavior in a water maze task. $\boldsymbol{A}$, Experimental design. $\boldsymbol{B}$, Representative images of DAPI staining and DCX + cells in the dentate gyrus of mice in the ablated and control groups. Scale bar: $150 \mu \mathrm{m}$. C, The densities of DCX+ cells. $\boldsymbol{D}$, The volume of the granule cell layer. $\boldsymbol{E}, \boldsymbol{F}$, Latency to locate the platform during pretraining $(\boldsymbol{E})$ and training $(\boldsymbol{F})$. The two groups improved performance similarly well in both pretraining and training. $\boldsymbol{G}$, The position of the removed platform and the area near the platform in probe trials. The area near the platform is defined as the circular area within $14 \mathrm{~cm}$ of the center of the former platform position. $\boldsymbol{H}$, Occupancy plots showing average time that mice spent at different positions in the pool. The color bar below shows the color code for occupancy time; warmer colors indicate high occupancy, while cooler colors represent low occupancy. Dotted circles indicate the area near the platform. I, Time spent in the area near the platform position, the number of platform entries and swimming speed in probe tests. A dotted line indicates the chance level in time spent near the platform position. $\boldsymbol{J}$, Latency to reach the position of the removed platform. $\boldsymbol{K}$, Percentage of time spent in the area near the platform position and the number of platform entries per $10 \mathrm{~s}$ after the first entries into the position of the removed platform. $L$, Duration per visit to the area near the platform. $\boldsymbol{M}$, Latency to locate the platform during re-training. The two groups improved performance similarly well; ${ }^{\star} p<0.05,{ }^{* *} p<0.01$.

hippocampus-dependent fear conditioning paradigm (Fig. 8A). $7 \mathrm{~d}$ after viral vector injection, the mice underwent training for contextual fear conditioning. The mice were moved to context $A$ and an electrical shock (2s) was given after $180 \mathrm{~s}$ (training). Next day, a shorter shock (0.2 s) was given as a reminder in the same context ("reminder" training), and then DT was systemically injected (ablated group, $n=18$ mice). After $7 \mathrm{~d}$, context test was conducted.
The control group ( $n=20$ mice) underwent the same protocol except they were injected with viral vector+PBS or PBS +DT. We confirmed a reduction of DCX + cells in the ablated group $\left(p=7 \times 10^{-6}, t_{(31.165)}=5.363\right.$, independent sample $t$ test; Fig. $8 B$ ), while the volume of the granule cell layer was intact $\left(p=0.768, t_{(36)}=-0.298\right.$, independent sample $t$ test; Fig. $8 C$ ). In training and reminder training (which were conducted before inducing the ablation), we 
A

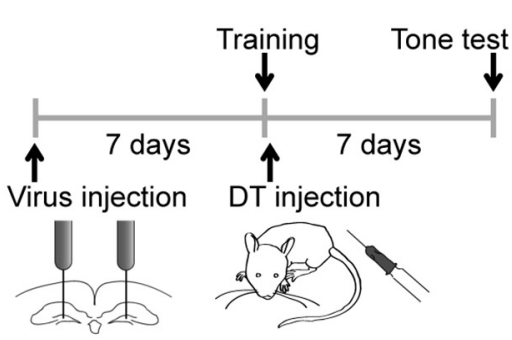

D

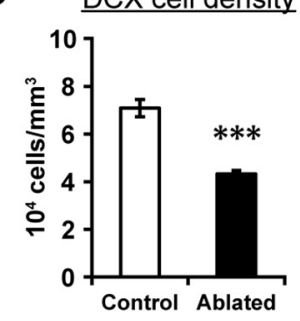

F Tone test

Context B

$5 \mathrm{x}$ Tone + No shock

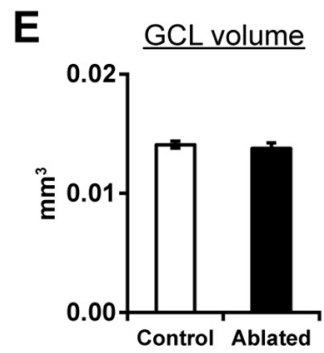

B Iraining

Context A

$5 x$ Tone + Shock

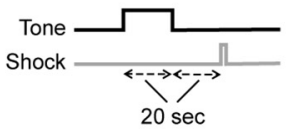

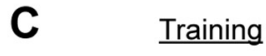

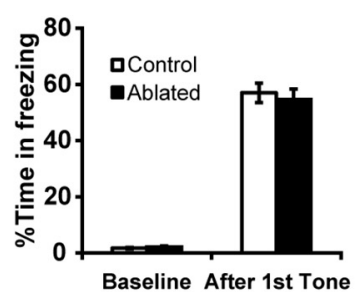

H

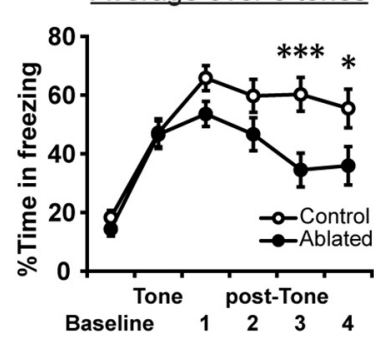

1st Tone only

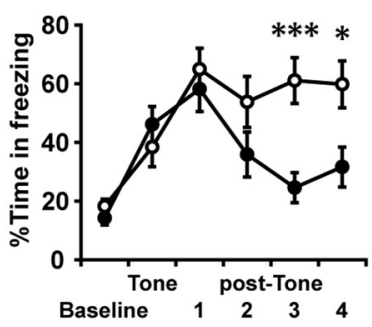

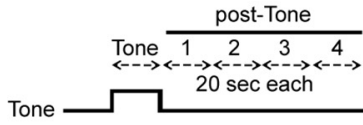

J
G

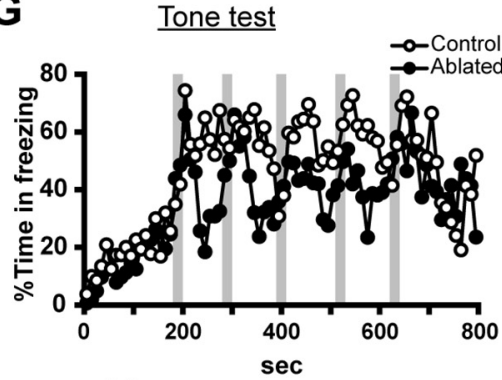

K
Freezing episode

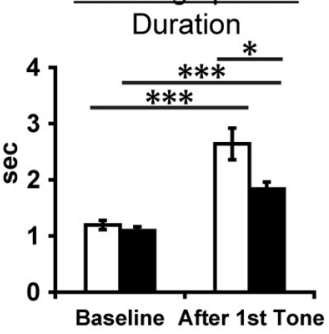

Figure 7. Posttraining ablation impaired the persistence of tone-induced freezing in a tone trace fear conditioning task. $\boldsymbol{A}$, Experimental design. $\boldsymbol{B}$. Timings of a tone (conditional stimulus) and an electrical shock (unconditioned stimulus) during training. $\boldsymbol{C}$, Percentage time in freezing during the baseline period and after the onset of first tone during training. No significant difference was detected between the groups. $\boldsymbol{D}$, The densities of DCX+ cells. $\boldsymbol{E}$, The volume of the granule cell layer. $\boldsymbol{F}$, The definition of the tone and posttone periods in the tone test. G, Percentage time in freezing for every $10 \mathrm{~s}$ during the tone test. Gray areas indicate the timing of tone deliveries. $\boldsymbol{H}, \boldsymbol{I}$, Percentage time in freezing during baseline, tone, posttone 1, 2, 3, and 4 periods, averaged over five tones $(\boldsymbol{H})$ or for first tone only $(\boldsymbol{I})$. $\boldsymbol{J}, \boldsymbol{K}$, Frequency $(\boldsymbol{J})$ and duration $(\boldsymbol{K})$ of freezing episodes during the baseline period and after the onset of first tone in the tone test; ${ }^{*} p<0.05,{ }^{\star \star *} p<0.005$.

did not find significant difference between the two groups in freezing level before or after the foot shock [baseline: $p=0.700, t_{(36)}=-0.389$, after shock: $p=0.568, t_{(36)}=0.576$ (Fig. 8D); baseline: $p=0.719, t_{(36)}=-0.363$, after shock: $p=0.455, t_{(36)}=0.755$, independent-sample $t$ tests (Fig. $8 E)$ ].

In the context test, overall freezing level was significantly lower in the ablated group compared with control $\left(p=0.046, t_{(33.9)}=-2.074\right.$, independent-sample $t$ test; Fig. $8 F, G$ ), indicating that the postlearning ablation impaired fear memory. Motivated from the previous two experiments showing impairment at late, but not early, time points in memory retrieval tests, we examined (1) latency for cumulative freezing duration to reach $20 \mathrm{~s}$; (2) percentage of time in freezing before reaching cumulative freezing duration of $20 \mathrm{~s}$; and (3) the same percentage after reaching cumulative freezing of $20 \mathrm{~s}$. Cumulative duration of $20 \mathrm{~s}$ is an arbitrary value which corresponds to $\sim 20 \%$ of mean total freezing time. We did not find significant group difference in the first two, which are the indexes of memory retrieval performance at early time points in the test $\left(p=0.622, t_{(36)}=-0.963\right.$ and $p=0.322, t_{(31.164)}=1.006$, independent-sample $t$ tests; Fig. $8 H, I)$. In contrast, we found a significant reduction in percentage time in freezing after cumulative freezing duration reached $20 \mathrm{~s}(p=0.033$, $t_{(36)}=2.216$, independent-sample $t$ test; Fig. 8J). We have also analyzed the number and duration of freezing episodes. Consistent with tone fear conditioning, the number of freezing episodes was not different between the groups $\left(p=0.285, t_{(36)}=-1.086\right.$, independent-sample $t$ test; Fig. $8 K)$, but their duration was significantly shorter in the ablated group than control $\left(p=0.028, t_{(27.742)}=2.323\right.$, independent-sample $t$ test). Thus, the persistence of learned fear responses to the context was impaired by the posttraining ablation.

\section{Discussion}

\section{Methodological consideration}

We established a novel method ablating DCX+ immature neurons in the dentate gyrus. Ablation is highly selective to immature neurons at the early maturational stages; 
A

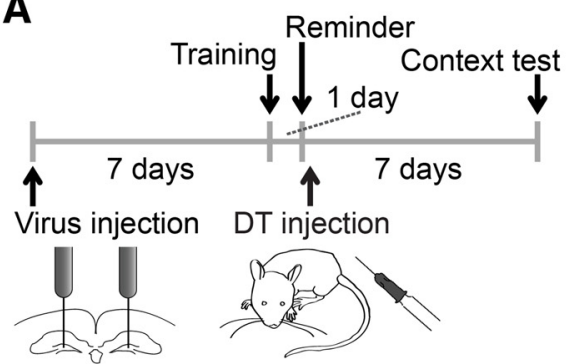

D

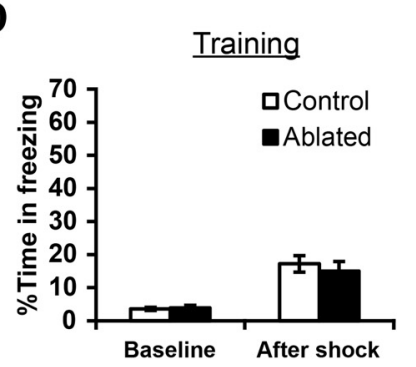

E
B

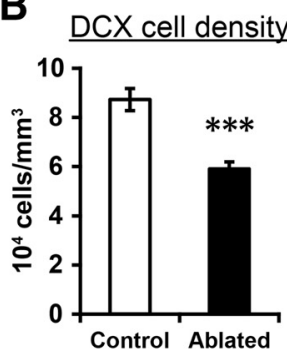

C

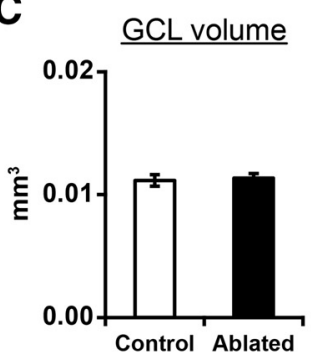

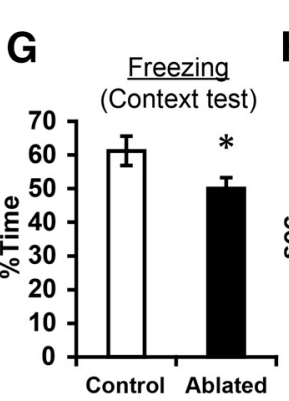
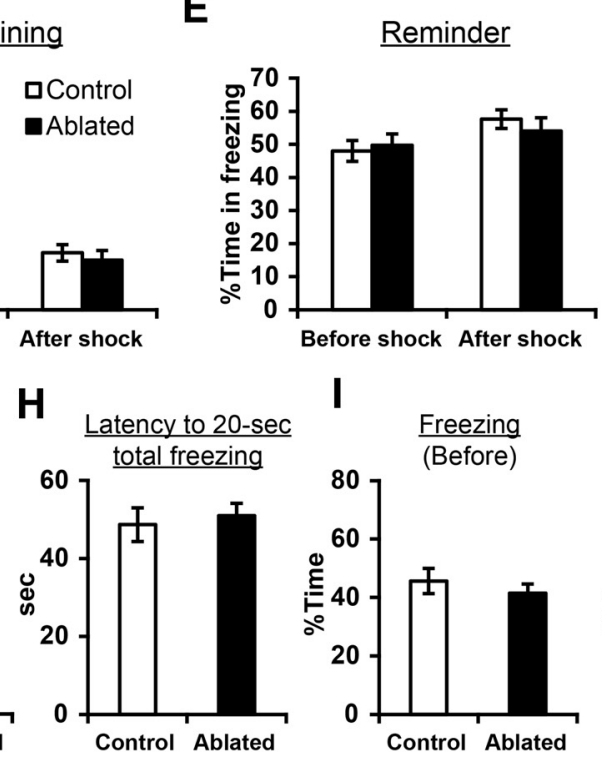

F
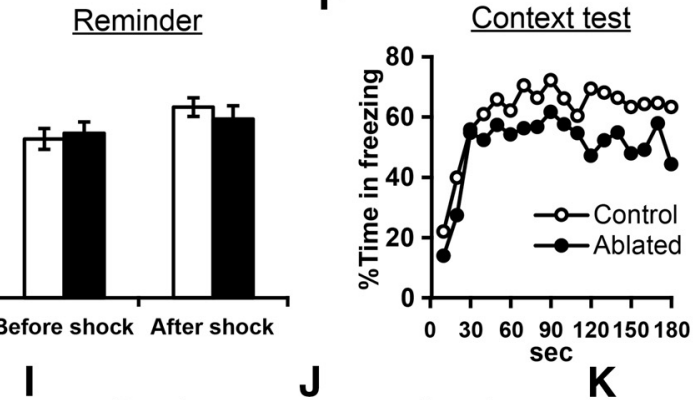

J

$\mathrm{K}$
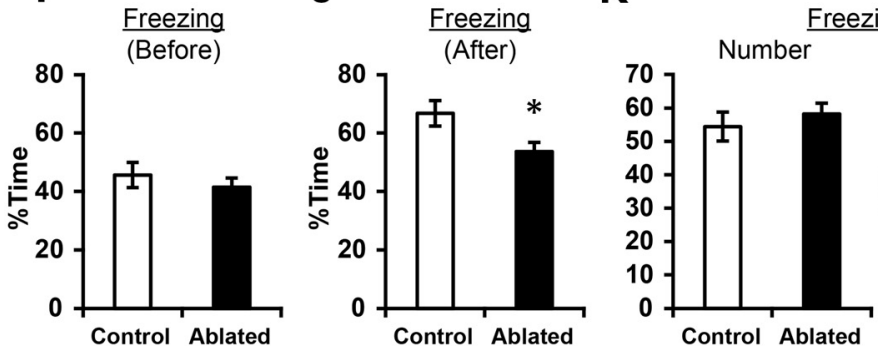

Freezing episode

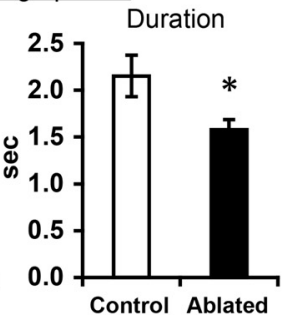

Figure 8. Posttraining ablation impaired the persistence of context-induced freezing in a contextual fear conditioning task. $\boldsymbol{A}$, Experimental design. $\boldsymbol{B}$, The densities of DCX+ cells. $\boldsymbol{C}$, The volume of the granule cell layer. $\boldsymbol{D}, \boldsymbol{E}$, Percentage time in freezing during the baseline period and after the shock in training $(\boldsymbol{D})$ and remainder training $(\boldsymbol{E})$. No significance different was detected between the groups. $\boldsymbol{F}$, Percentage time in freezing for every $10 \mathrm{~s}$ during the context test. $\boldsymbol{G}$, Percentage time in freezing in the context test. $\boldsymbol{H}$, Latency to reach 20 -s cumulative freezing in the context test. $\boldsymbol{I}, \boldsymbol{J}$, Percentage time in freezing before $(\boldsymbol{I})$ or after $(\boldsymbol{J})$ reaching cumulative freezing of $20 \mathrm{~s}$. $\boldsymbol{K}$, The number and duration of freezing episodes; ${ }^{*} p<0.05,{ }^{* \star *} p<0.005$.

$>90$ and $>95 \%$ of ablated neurons are estimated to beless than three and less than four weeks old, respectively. However, the method causes a side effect of inflammation-like response detected by increase in Iba1+ cells. Similar inflammation-like response has been also observed in previous studies that used different methods involving cell ablation (Arruda-Carvalho et al., 2011; Seo et al., 2015). Inflammation is known to cause damages in neurons and compromise their functions (DiSabato et al., 2016). Therefore, we cannot distinguish whether the observed behavioral change is because of inflammation or ablation of immature neurons. We have evaluated a sign of detrimental effects on other neuronal populations than DCX + immature neurons in the dentate gyrus. However, we did not detect any structural sign of damages in granule cell layer volume, the density, dendritic complexity and spine density of granule cells. Functionally, we did not detect a clear change in c-fos expression, a proxy of neuronal activation. Intact memory performance in re-training for new platform positions after ablation suggests that the overall function of dentate gyrus is intact (Sutherland et al., 1983; Nanry et al., 1989).
Main finding: impairment in within-trial persistence of learned behavior and its dissociation from preserved performance in the initial phase of retrieval tests

In this study, we showed that the postlearning ablation results in behavioral deficits during memory retrieval tests of three hippocampus-dependent memory tasks. In these widely used retrieval tests, animals respond to cues (i.e., visual cues, context, tone) and show learned behavior (platform search and freezing). However, the learned behavior is not reinforced because of the absence of the platform or electrical shocks. Although the absence of reinforcement eventually leads to reduction/cessation of learned behavior [so called "extinction" or "within-session extinction" (Myers and Davis, 2002). In this context, in the meaning at the behavioral level as often used in psychology], animals continue to show the learned behavior for some duration, which we term as "persistence of learned behavior" (note that we use the term "persistence" as a description of behavior but not cognitive ability/tendency). We observed that the postlearning ablation impairs such persistence of learned behavior, which contains two common features. First, the ablated group showed intact 
Table 1: Statistical results for Figure 6

\begin{tabular}{|c|c|c|c|c|c|c|}
\hline \multirow{2}{*}{\multicolumn{7}{|c|}{$\begin{array}{c}\text { Pretraining (three-way, group } \times \text { trial } \times \text { day, control, } n=18 \text { mice; ablated, } n=18 \text { mice) } \\
\text { Latency to platform }\end{array}$}} \\
\hline & & & & & & \\
\hline Day $\times$ group & 0.858 & 3,102 & 0.254 & 0.630 & $2.434,82.753$ & 0.524 \\
\hline Trial & $1.4 \times 10^{-11}$ & $1.529,51.982$ & 52.033 & 0.006 & 2,68 & 5.494 \\
\hline Trial $\times$ group & 0.885 & $1.529,51.982$ & 0.072 & 0.612 & 2,68 & 0.495 \\
\hline Group & 0.870 & 1,34 & 0.027 & 0.157 & 1,34 & 2.089 \\
\hline
\end{tabular}

Training (two-way, block $\times$ group, control, $n=18$ mice; ablated, $n=18$ mice)

\begin{tabular}{|c|c|c|c|c|c|c|}
\hline & \multicolumn{3}{|c|}{ Latency to platform } & \multicolumn{3}{|c|}{ Speed } \\
\hline & $p$ & df & $F$ & $p$ & df & $F$ \\
\hline Block & $2.6 \times 10^{-12}$ & $4.313,146.653$ & 17.434 & $7.8 \times 10^{-5}$ & $5.291,179.911$ & 5.440 \\
\hline Block $\times$ group & 0.321 & $4.313,146.653$ & 1.184 & 0.474 & $5.291,179.911$ & 0.919 \\
\hline Group & 0.566 & 1,34 & 0.336 & 0.533 & 1,34 & 0.398 \\
\hline
\end{tabular}

Re-training (three-way, group $\times$ trial $\times$ day, control, $n=18$ mice; ablated, $n=18$ mice)

\begin{tabular}{|c|c|c|c|c|c|c|}
\hline & \multicolumn{3}{|c|}{ Latency to platform } & \multicolumn{3}{|c|}{ Speed } \\
\hline Day & 0.034 & 2,68 & 3.565 & 0.065 & $1.682,57.174$ & 3.020 \\
\hline Trial & $1.7 \times 10^{-30}$ & $5.849,198.874$ & 38.729 & 0.0002 & 7,238 & 4.199 \\
\hline Trial $\times$ group & 0.756 & $5.849,198.874$ & 0.562 & 0.494 & 7,238 & 0.917 \\
\hline Day $\times$ trial & 0.478 & $9.888,336.198$ & 0.960 & 0.127 & $12.302,418.255$ & 1.479 \\
\hline Day $\times$ trial $\times$ group & 0.871 & $9.888,336.198$ & 0.524 & 0.627 & $12.302,418.255$ & 0.826 \\
\hline
\end{tabular}

performance of learned behavior at early time points during the memory retrieval tests. However, later in the tests, the ablated group reduced learned behavior compared with control. Second, when we examined the duration of individual episodes of learned behavior (platform search and freezing), their durations were reduced in the ablated group. Both of these common features point to impairment in persistence of learned behavior, although they are different in their timescales, over the entire trials and for individual behavioral episodes. These effects were consistently observed in three hippocampus-dependent memory tasks.

We found that the effect of the postlearning ablation showed dissociation between initial performance and persistence of learned behavior; the ablation impaired persistence while preserving initial performance intact. In typical studies, it is rare for these measurements to be considered separately in memory retrieval tests, and rather expected to be strongly correlated with each other. Although previous studies separated different behavioral components in a single type of tasks (Dalm et al., 2000; Korz, 2006; Maei et al., 2009), it is rare to make an attempt to find such dissociable behavioral components that are common across different types of hippocampus-dependent memory tasks with different behavioral and cognitive demands. We have found the dissociation consistently in the three hippocampus-dependent tasks.

The observed dissociation effect indicates that there are at least two separate components of behavior during the memory retrieval tests. These two components, initial performance and persistence, are at least partially independent and dissociable in the sense that the latter can be high or low even if the former is similarly well. Furthermore, the dissociated effect suggests that two separate brain functions and their underlying mechanisms govern learned behavior in the memory retrieval tests. One function supports persistence of learned behavior, which was impaired by the ablation. The other supports initial expression of learned behavior, which was not affected by the postlearning ablation.

\section{Speculating about relationship between persistence of learned behavior and memory strength}

In this section, we speculate about relationship between persistence of learned behavior and memory. In typical behavioral studies on mechanisms underlying memory, one interprets a decrease in expression of learned behavior caused by an experimental manipulation as weakening/impairment of memory while an increase as strengthening/improvement of memory. We found that our postlearning manipulation impairs performance specifically at late time points of memory retrieval tests, but not that at early time points. Applying the same interpretation method as above, we could interpret our finding as an indication that the postlearning manipulation impairs the aspect of memory strength that regulates behavior at late time points while sparing another aspect of memory strength that influences behavior at early time points. This 
interpretation of dissociated effects between the early and late time points also hints at or assumes an idea that there may be two separate aspects of memory strength supporting learned behavior at different time points, which have separate underlying mechanisms.

Expression of learned behavior is thought to depend on memory storage and retrieval. For example, recent studies indicated that amnesia induced by protein-synthesis inhibitor or in mouse models of Alzheimer's disease is because of compromised memory retrieval but not storage (Ryan et al., 2015; Roy et al., 2016), which may indicate that the strength/effectiveness of these two processes may be independent and separable. Bjork and Bjork postulated two types of memory strength (Bjork and Bjork, 1992); one refers to how easily the learned information is retrieved while the other represents how well/detailed learned information is retained. The latter may be related to vividness of or subjective certainty about a memory, which could influence persistence of learned behavior. Imagine that you vividly remember that you stored your room key in a drawer of a cabinet last night and you are $100 \%$ sure about it. Even if your initial search attempt failed to find the key in the drawer, you would continue to search the key for a long time. On the other hand, if you are not sure about where you stored the key and happen to search the drawer, you would stop it more quickly and start searching other locations. Studies have showed that human subjects made a more persistent effort in searching a missing object or recalling a difficult-to-be-retrieved memory when they were more certain about the memories (Gruneberg et al., 1977; DeLoache and Brown, 1984). Although we cannot tell what exactly is reflected by the mouse behavior, it would be reasonable to speculate that initial performance and persistence of learned behavior in memory retrieval tests reflect different aspects of memory in terms of how well a mouse remember the memory.

So far in this section, we discussed that persistence of learned behavior may reflect memory strength, which is a characteristic of individual memories. However, an alternative way to explain our observation is that ablation impaired subject's cognitive ability/tendency of translating learned information into long-lasting behavior within a trial. For example, cognitive stability is the ability to keep one's perspective stable without being affected by environmental changes/distractions (Ueltzhöffer et al., 2015; Dreisbach and Fröber, 2019; Uddin, 2021), in other words, the ability to suppress cognitive flexibility or, in memory-related contexts, the ability to keep long-lasting working memory. Disruption of within-trial cognitive stability would result in premature cessation of subject's perspective based on learned information, which can explain our observation of reduction in learned behavior at late phases in memory retrieval tests.

How can we distinguish whether observed changes in within-trial persistence of learned behavior represents impairment in specific memories or cognitive ability/tendency? If impairment is specific to a subset of memories, we would observe changes in within-trial persistence for some memories but not for others. On the other hand, if impairment is in cognitive ability/tendency, we would observe changes in within-trial persistence similarly for all memories. Starting from $1 \mathrm{~d}$ after the water maze probe tests, we performed retraining for $3 \mathrm{~d}$ while changing platform location each day. By the end of days 1 and 2, mice would have learned new platform locations well. In the next trials (the first trials of days 2 and 3 , respectively), mice experienced similar situations to probe tests where the platform was not located at the newly learned locations. In these trials, how persistently mice continue to search the former platform positions (learned on days 1 and 2 , respectively) would delay mice to reach the platform in a new position. If cognitive ability/tendency was affected, we should have seen a change in performance in these trials. If impairment was specific to the memory for original platform position during training, such a change would not be expected. Our result is consistent with the latter possibility, although our retraining experiment may not necessarily be optimized to address this question and we need further investigation to reach a firm conclusion. In addition, distinction between the possibilities may not be straightforward purely based on behavioral observation and would require further studies involving different techniques.

\section{Potential involvement of DCX + immature neurons in persistence of learned behavior}

We observed that the postlearning ablation of immature neurons caused inflammation-like response and therefore are not able to attribute the observed impairment in persistence to the ablation of DCX+ immature neurons. Nonetheless, we believe it is worth discussing existing literature both consistent and seemingly conflicting with our results from the viewpoint of a potential postlearning role of immature neurons. First, previous studies demonstrated a role of immature neurons at the early maturational stage in memory by removing immature neurons before memory formation (Deng et al., 2009; Seo et al., 2015). These findings are consistent with our finding, but do not differentiate whether those immature neurons are involved in initial memory acquisition only or later memory processes because they blocked adult neurogenesis before memory acquisition. Second, other studies found memory impairment when ablating adult-born neurons (Arruda-Carvalho et al., 2011; Suárez-Pereira et al., 2015) or silencing them (Kumar et al., 2020) after training for hippocampus-dependent tasks. These findings are consistent with our finding in that new neurons have a role beyond initial memory acquisition, although they did not reveal the age of new neurons involved in this role. Third, using optogenetic silencing during memory retrieval tests, $\mathrm{Gu}$ et al. (2012) indicated that the postlearning role is mediated by new neurons at later maturational stages, but failed to detect impairment when DCX+ immature neurons was silenced during memory retrieval tests. At a quick look, one may find this finding conflicting with ours. However, they performed optogenetic silencing only during memory retrieval tests while ablation in our method was induced immediately after the completion of training. 
Therefore, it is possible that the existence of new neurons at early maturational periods is required during memory retention period but not during memory retrieval. Fourth, Vukovic et al. (2013) performed a conceptually similar experiment involving immature neuron ablation using an active place avoidance task. This study did not find impaired performance in retrieval test trials when immature neurons were ablated after normal acquisition of a place avoidance task. An important difference from our study in the experimental design is the presence of reinforcement during memory retrieval tests. During training for the active place avoidance task, mice learned to stay in a safe zone to avoid noxious electrical shocks; otherwise, they received electrical shocks when they went out of the safe zone. This reinforcing design was kept same in the memory retrieval tests, so that the learned behavior continued to be reinforced. Therefore, the retrieval test itself supported persistence of learned behavior and may have masked the contribution of $\mathrm{DCX}+$ immature neurons being reflected to persistent behavior. This may be why this previous study did not find an effect after postlearning ablation of DCX + immature neurons.

New neurons at the early immature stage are in the process of constructing new circuits by extending neurites, forming synapses and making survival-or-death decisions (Biebl et al., 2000; Zhao et al., 2006). These phenomena are experience/activity dependent (Ge et al., 2006; OverstreetWadiche et al., 2006; Tashiro et al., 2006, 2007; Tronel et al., 2010; Chancey et al., 2013; Aasebø et al., 2018). Further, although long-term potentiation is more easily induced in immature neurons at the early stage than in mature neurons (Wang et al., 2000; Snyder et al., 2001; Schmidt-Hieber et al., 2004), the extent of the potentiation is smaller in younger neurons than in their older counterparts (Ge et al., 2007). These characteristics seem to better support gradual changes which do not complete immediately during memory acquisition but accumulate over time. Through these slow circuit modification processes, DCX + immature neurons may influence how acquired memory supports the expression of learned behavior and therefore their presence during memory retention period is required to form strong memory that leads to persistent learned behavior. A further study using a postlearning manipulation without inflammatory and other side effects would be required to determine whether DCX + immature neurons have a postlearning role in persistence of learned behavior.

In conclusion, our work revealed that there are dissociable behavioral components during memory retrieval tests of hippocampus-dependent memory tasks, which have been widely used in rodents. These two behavioral components, initial performance and persistence of learned behavior, are likely to reflect different brain functions and be mediated by separate mechanisms, which might represent different modalities of memory strength. These simple dissociable measurements in widely used behavioral paradigms would be useful to understand the detailed mechanisms underlying the expression of learned behavior and potentially different modalities of memory strength in mice.

\section{References}

Aasebø IE, Blankvoort S, Tashiro A (2011) Critical maturational period of new neurons in adult dentate gyrus for their involvement in memory formation. Eur J Neurosci 33:1094-1100.

Aasebø IEJ, Kasture AS, Passeggeri M, Tashiro A (2018) A behavioral task with more opportunities for memory acquisition promotes the survival of new neurons in the adult dentate gyrus. Sci Rep 8:7369.

Abusaad I, MacKay D, Zhao J, Stanford P, Collier DA, Everall IP (1999) Stereological estimation of the total number of neurons in the murine hippocampus using the optical disector. J Comp Neurol 408:560-566.

Åmellem I, Suresh S, Chang CC, Tok SSL, Tashiro A (2017) A critical period for antidepressant-induced acceleration of neuronal maturation in adult dentate gyrus. Transl Psychiatry 7:e1235.

Andersen JB, Sternberg C, Poulsen LK, Bjorn SP, Givskov M, Molin S (1998) New unstable variants of green fluorescent protein for studies of transient gene expression in bacteria. Appl Environ Microbiol 64:2240-2246.

Arruda-Carvalho M, Sakaguchi M, Akers KG, Josselyn SA, Frankland PW (2011) Posttraining ablation of adult-generated neurons degrades previously acquired memories. J Neurosci 31:1511315127.

Biebl M, Cooper CM, Winkler J, Kuhn HG (2000) Analysis of neurogenesis and programmed cell death reveals a self-renewing capacity in the adult rat brain. Neurosci Lett 291:17-20.

Bjork RA, Bjork EL (1992) A new theory of disuse and an old theory of stimulus fluctuation. In From learning processes to cognitive processes: essays in honor of William K. Estes (Healy SKA and Shiffrin R, eds), pp 235-267. Hillsdale: Erlbaum.

Brown JP, Couillard-Després S, Cooper-Kuhn CM, Winkler J, Aigner L, Kuhn HG (2003) Transient expression of doublecortin during adult neurogenesis. J Comp Neurol 467:1-10.

Buch T, Heppner FL, Tertilt C, Heinen TJAJ, Kremer M, Wunderlich FT, Jung S, Waisman A (2005) A Cre-inducible diphtheria toxin receptor mediates cell lineage ablation after toxin administration. Nat Methods 2:419-426.

Chancey JH, Adlaf EW, Sapp MC, Pugh PC, Wadiche JI, OverstreetWadiche LS (2013) GABA depolarization is required for experience-dependent synapse unsilencing in adult-born neurons. J Neurosci 33:6614-6622.

Collier RJ (1975) Diphtheria toxin: mode of action and structure. Bacteriol Rev 39:54-85.

Crawley JN (2000) What's wrong with my mouse? :behavioral phenotyping of transgenic and knockout mice. New York: Wiley.

Dalm S, Grootendorst J, de Kloet ER, Oitzl MS (2000) Quantification of swim patterns in the Morris water maze. Behav Res Methods Instrum Comput 32:134-139.

DeLoache JS, Brown AL (1984) Where do I go next? Intelligent searching by very young children. Dev Psychol 20:37-44.

Deng W, Saxe MD, Gallina IS, Gage FH (2009) Adult-born hippocampal dentate granule cells undergoing maturation modulate learning and memory in the brain. J Neurosci 29:13532-13542.

Denny CA, Burghardt NS, Schachter DM, Hen R, Drew MR (2012) 4to 6-week-old adult-born hippocampal neurons influence noveltyevoked exploration and contextual fear conditioning. Hippocampus 22:1188-1201.

DiSabato DJ, Quan N, Godbout JP (2016) Neuroinflammation: the devil is in the details. J Neurochem 139 Suppl 2:136-153.

Dorland RB, Middlebrook JL, Leppla SH (1979) Receptor-mediated internalization and degradation of diphtheria toxin by monkey kidney cells. J Biol Chem 254:11337-11342.

Dreisbach G, Fröber K (2019) On how to be flexible (or not): modulation of the stability-flexibility balance. Curr Dir Psychol Sci 28:3-9.

Espósito MS, Piatti VC, Laplagne DA, Morgenstern NA, Ferrari CC, Pitossi FJ, Schinder AF (2005) Neuronal differentiation in the adult hippocampus recapitulates embryonic development. J Neurosci 25:10074-10086.

Gage FH (2000) Mammalian neural stem cells. Science 287:14331438. 
Ge S, Goh EL, Sailor KA, Kitabatake Y, Ming GL, Song H (2006) GABA regulates synaptic integration of newly generated neurons in the adult brain. Nature 439:589-593.

Ge S, Yang CH, Hsu KS, Ming GL, Song H (2007) A critical period for enhanced synaptic plasticity in newly generated neurons of the adult brain. Neuron 54:559-566.

Geib BR, Stanley ML, Wing EA, Laurienti PJ, Cabeza R (2017) Hippocampal contributions to the large-scale episodic memory network predict vivid visual memories. Cereb Cortex 27:680-693.

Gruneberg MM, Monks J, Sykes RN (1977) Some methodological problems with feeling of knowing studies. Acta Psychologica 41:365-371.

Gu Y, Arruda-Carvalho M, Wang J, Janoschka SR, Josselyn SA, Frankland PW, Ge S (2012) Optical controlling reveals time-dependent roles for adult-born dentate granule cells. Nat Neurosci 15:1700-1706.

Iwamoto R, Higashiyama S, Mitamura T, Taniguchi N, Klagsbrun M, Mekada E (1994) Heparin-binding EGF-like growth factor, which acts as the diphtheria toxin receptor, forms a complex with membrane protein DRAP27/CD9, which up-regulates functional receptors and diphtheria toxin sensitivity. EMBO $\mathrm{J}$ 13:2322-2330.

Jagasia R, Steib K, Englberger E, Herold S, Faus-Kessler T, Saxe M, Gage FH, Song H, Lie DC (2009) GABA-cAMP response elementbinding protein signaling regulates maturation and survival of newly generated neurons in the adult hippocampus. J Neurosci 29:7966-7977.

Karl C, Couillard-Despres S, Prang P, Munding M, Kilb W, Brigadski T, Plötz S, Mages W, Luhmann H, Winkler J, Bogdahn U, Aigner L (2005) Neuronal precursor-specific activity of a human doublecortin regulatory sequence. J Neurochem 92:264-282.

Kempermann G, Gast D, Kronenberg G, Yamaguchi M, Gage FH (2003) Early determination and long-term persistence of adultgenerated new neurons in the hippocampus of mice. Development 130:391-399.

Korz V (2006) Water maze swim path analysis based on tracking coordinates. Behav Res Methods 38:522-528.

Kumar D, Koyanagi I, Carrier-Ruiz A, Vergara P, Srinivasan S, Sugaya Y, Kasuya M, Yu TS, Vogt KE, Muratani M, Ohnishi T, Singh S, Teixeira CM, Chérasse Y, Naoi T, Wang SH, Nondhalee P, Osman BAH, Kaneko N, Sawamoto K, et al. (2020) Sparse Activity of Hippocampal Adult-Born Neurons during REM Sleep Is Necessary for Memory Consolidation. Neuron 107:552-565. e10.

Lacy JW, Stark CEL (2013) The neuroscience of memory: implications for the courtroom. Nat Rev Neurosci 14:649-658.

Maei HR, Zaslavsky K, Teixeira CM, Frankland PW (2009) What is the most sensitive measure of water maze probe test performance? Front Integr Neurosci 3:4.

Maren S (2001) Neurobiology of Pavlovian fear conditioning. Annu Rev Neurosci 24:897-931.

Matzel LD, Han YR, Grossman H, Karnik MS, Patel D, Scott N, Specht SM, Gandhi CC (2003) Individual differences in the expression of a "general" learning ability in mice. J Neurosci 23:64236433.

Morris RG, Garrud P, Rawlins JN, O'Keefe J (1982) Place navigation impaired in rats with hippocampal lesions. Nature 297:681-683.

Myatt DR, Hadlington T, Ascoli GA, Nasuto SJ (2012) Neuromantic from semi-manual to semi-automatic reconstruction of neuron morphology. Front Neuroinform 6:4.

Myers KM, Davis M (2002) Behavioral and neural analysis of extinction. Neuron 36:567-584.

Nanry KP, Mundy WR, Tilson HA (1989) Colchicine-induced alterations of reference memory in rats: role of spatial versus non-spatial task components. Behav Brain Res 35:45-53.

Overstreet-Wadiche LS, Bromberg DA, Bensen AL, Westbrook GL (2006) Seizures accelerate functional integration of adult-generated granule cells. J Neurosci 26:4095-4103.
Qin S, van Marle HJF, Hermans EJ, Fernández G (2011) Subjective sense of memory strength and the objective amount of information accurately remembered are related to distinct neural correlates at encoding. J Neurosci 31:8920-8927.

Roy DS, Arons A, Mitchell TI, Pignatelli M, Ryan TJ, Tonegawa S (2016) Memory retrieval by activating engram cells in mouse models of early Alzheimer's disease. Nature 531:508-512.

Ryan TJ, Roy DS, Pignatelli M, Arons A, Tonegawa S (2015) Memory. Engram cells retain memory under retrograde amnesia. Science 348:1007-1013.

Schindelin J, Arganda-Carreras I, Frise E, Kaynig V, Longair M, Pietzsch T, Preibisch S, Rueden C, Saalfeld S, Schmid B, Tinevez JY, White DJ, Hartenstein V, Eliceiri K, Tomancak P, Cardona A (2012) Fiji: an open-source platform for biological-image analysis. Nat Methods 9:676-682.

Schmidt-Hieber C, Jonas P, Bischofberger J (2004) Enhanced synaptic plasticity in newly generated granule cells of the adult hippocampus. Nature 429:184-187.

Sekeres MJ, Winocur G, Moscovitch M (2018) The hippocampus and related neocortical structures in memory transformation. Neurosci Lett 680:39-53.

Seo DO, Carillo MA, Chih-Hsiung Lim S, Tanaka KF, Drew MR (2015) Adult hippocampal neurogenesis modulates fear learning through associative and nonassociative mechanisms. J Neurosci 35:11330-11345.

Shors TJ, Miesegaes G, Beylin A, Zhao M, Rydel T, Gould E (2001) Neurogenesis in the adult is involved in the formation of trace memories. Nature 410:372-376.

Snyder JS, Kee N, Wojtowicz JM (2001) Effects of adult neurogenesis on synaptic plasticity in the rat dentate gyrus. J Neurophysiol 85:2423-2431.

Snyder JS, Hong NS, McDonald RJ, Wojtowicz JM (2005) A role for adult neurogenesis in spatial long-term memory. Neuroscience 130:843-852.

Snyder JS, Choe JS, Clifford MA, Jeurling SI, Hurley P, Brown A, Kamhi JF, Cameron HA (2009) Adult-born hippocampal neurons are more numerous, faster maturing, and more involved in behavior in rats than in mice. J Neurosci 29:1448414495.

Squire LR, Wixted JT, Clark RE (2007) Recognition memory and the medial temporal lobe: a new perspective. Nat Rev Neurosci 8:872883.

Suárez-Pereira I, Canals S, Carrión AM (2015) Adult newborn neurons are involved in learning acquisition and long-term memory formation: the distinct demands on temporal neurogenesis of different cognitive tasks. Hippocampus 25:51-61.

Sutherland RJ, Whishaw IQ, Kolb B (1983) A behavioural analysis of spatial localization following electrolytic, kainate- or colchicine-induced damage to the hippocampal formation in the rat. Behav Brain Res 7:133-153.

Tashiro A, Sandler VM, Toni N, Zhao C, Gage FH (2006) NMDA-receptor-mediated, cell-specific integration of new neurons in adult dentate gyrus. Nature 442:929-933.

Tashiro A, Makino H, Gage FH (2007) Experience-specific functional modification of the dentate gyrus through adult neurogenesis: a critical period during an immature stage. J Neurosci 27:32523259.

Tashiro A, Zhao C, Suh H, Gage FH (2015a) Imaging newborn granule cells in fixed sections. Cold Spring Harb Protoc 2015:932-933.

Tashiro A, Zhao C, Suh H, Gage FH (2015b) Purification and injection of retroviral vectors. Cold Spring Harb Protoc 2015:925931.

Tronel S, Fabre A, Charrier V, Oliet SH, Gage FH, Abrous DN (2010) Spatial learning sculpts the dendritic arbor of adult-born hippocampal neurons. Proc Natl Acad Sci USA 107:79637968.

Trouche S, Bontempi B, Roullet P, Rampon C (2009) Recruitment of adult-generated neurons into functional hippocampal networks 
contributes to updating and strengthening of spatial memory. Proc Natl Acad Sci USA 106:5919-5924.

Uddin LQ (2021) Cognitive and behavioural flexibility: neural mechanisms and clinical considerations. Nat Rev Neurosci 22:167-179.

Ueltzhöffer K, Armbruster-Genç DJN, Fiebach CJ (2015) Stochastic dynamics underlying cognitive stability and flexibility. PLoS Comput Biol 11:e1004331.

Vukovic J, Borlikova GG, Ruitenberg MJ, Robinson GJ, Sullivan RK, Walker TL, Bartlett PF (2013) Immature doublecortin-positive hippocampal neurons are important for learning but not for remembering. J Neurosci 33:6603-6613.

Wang S, Scott BW, Wojtowicz JM (2000) Heterogenous properties of dentate granule neurons in the adult rat. J Neurobiol 42:248-257.

Wozniak PA, Gorzelanczyk EJ, Murakowski JA (1995) Two components of long-term memory. Acta Neurobiol Exp (Wars) 55:301-305.

Zhao C, Teng EM, Summers RG Jr, Ming GL, Gage FH (2006) Distinct morphological stages of dentate granule neuron maturation in the adult mouse hippocampus. J Neurosci 26:3-11. 